\title{
Élaboration d'un cadre de référence pour l'étude des stratégies : analyse de l'activité et étude de cas multiples dans deux usines de crabe
}

Development of a frame of reference for studying strategies: Activity analysis and the study of multiple cases in two crab plants

Elaboración de un marco de referencia para el estudio de las estrategias :

Análisis de la actividad y estudio de casos múltiples en dos plantas de

tratamiento del cangrejo

Marie-Ève Major et Nicole Vézina

\section{(2) OpenEdition}

Journals

\section{Édition électronique}

URL : http://journals.openedition.org/pistes/1843

DOI : 10.4000/pistes. 1843

ISSN : 1481-9384

Éditeur

Les Amis de PISTES

Édition imprimée

Date de publication : 1 novembre 2011

\section{Référence électronique}

Marie-Ėve Major et Nicole Vézina, «Élaboration d'un cadre de référence pour l'étude des stratégies

analyse de l'activité et étude de cas multiples dans deux usines de crabe », Perspectives

interdisciplinaires sur le travail et la santé [En ligne], 13-2 | 2011, mis en ligne le 01 novembre 2011,

consulté le 19 avril 2019. URL : http://journals.openedition.org/pistes/1843; DOI : 10.4000/

pistes. 1843

Ce document a été généré automatiquement le 19 avril 2019.

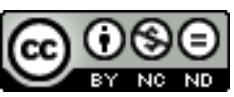

Pistes est mis à disposition selon les termes de la licence Creative Commons Attribution - Pas d'Utilisation Commerciale - Pas de Modification 4.0 International. 


\section{Élaboration d'un cadre de référence pour l'étude des stratégies : analyse de l'activité et étude de cas multiples dans deux usines de crabe}

Development of a frame of reference for studying strategies: Activity analysis and the study of multiple cases in two crab plants

Elaboración de un marco de referencia para el estudio de las estrategias : Análisis de la actividad y estudio de casos múltiples en dos plantas de tratamiento del cangrejo

Marie-Ève Major et Nicole Vézina

\section{Introduction}

1 Le secteur agroalimentaire fait depuis longtemps l'objet d'investigations dans le but de favoriser la prévention des troubles musculo-squelettiques (TMS) (Armstrong et coll., 1982 ; Mergler et coll., 1983, etc.). Les recherches ont cependant surtout été menées dans l'industrie de l'abattage et de la transformation de la viande et de la volaille (Toulouse et coll., 1995 ; Vézina et coll., 1995 ; Richard, 1997 ; Coutarel et coll., 2003 ; Ouellet et Vézina, 2008) alors que les recherches dans l'industrie des produits de la mer sont rares. Les quelques recherches dans ce secteur se sont davantage intéressées à l'industrie de la transformation du poisson en exploitation toute l'année plutôt qu'à celle des crustacés (crabe, crevette, homard) à opération saisonnière. Pourtant, ce secteur d'activité a connu une croissance importante après le moratoire sur la pêche à la morue à Terre-Neuve en 1992 (Schrank, 2005) où bon nombre de travailleurs de l'industrie de la pêche ont obtenu un emploi dans le secteur des mollusques et crustacés.

2 Les recherches réalisées dans l'industrie de la transformation du poisson font ressortir des risques élevés de TMS au cou et aux membres supérieurs (Chiang et coll., 1993; 
Ohlsson et coll., 1994), et ce, en particulier chez les travailleuses (Messing et Reveret, 1983 ; Nordander et coll., 1999). À notre connaissance, les quelques recherches effectuées sur les TMS dans l'industrie de la transformation du crabe ont été réalisées par des chercheurs du programme SafetyNet de l'Université Memorial à Terre-Neuve, une alliance communautaire pour la recherche en santé et en sécurité du travail des secteurs maritimes et côtiers de l'Atlantique Canada. L'un des projets du programme SafetyNet portait sur les TMS des travailleurs d'une usine de transformation du crabe située à Terre-Neuve (Solberg et coll., 2005). Dans cette étude, sur les 107 travailleurs ayant répondu au questionnaire (73 femmes, 34 hommes), $88 \%$ des répondantes et $82 \%$ des répondants ont mentionné souffrir de symptômes de TMS. Les régions corporelles les plus rapportées étaient les épaules, surtout chez les femmes, et les mains.

La plupart des travaux ayant porté sur la santé au travail au sein de l'industrie du crabe se sont attardés à l'asthme professionnel. Les travaux de Howse et collaborateurs (2006) ainsi que de Gautrin et collaborateurs (2010) présentent les résultats d'une étude de trois ans portant sur l'asthme et l'allergie professionnels au crabe au sein de quatre usines de la province de Terre-Neuve-Labrador au Canada. Les évaluations de santé ont été menées auprès de 215 travailleurs et des entretiens ont été réalisés auprès de 27 participants ( 24 femmes, 3 hommes) étant considérés comme à risque ou possiblement atteints d'asthme et d'allergie professionnels au crabe. D'après leurs résultats, les travailleurs et travailleuses éviteraient de présenter des réclamations à leur commission de la santé et de la sécurité au travail (WHSCC) dans le but de réduire les conséquences économiques possibles de leur maladie. L'incertitude des emplois en régions éloignées, les minces possibilités d'emplois de rechange pour les femmes (Neis, 1994), ainsi que la non-prise en compte des heures passées à recevoir des indemnités de compensation pour l'éligibilité à l'assurance-emploi (Howse et coll., 2006) inciteraient les travailleurs-euses de l'industrie de la transformation du crabe à demeurer au travail en dépit de leur maladie et de gérer eux-mêmes leur maladie. L'ensemble des travailleurs-euses questionnés dans l'étude de Howse et coll. (2006) ont mentionné avoir recours à des stratégies pour réduire ou prévenir les symptômes de l'asthme professionnel au travail. Les exemples de stratégies mentionnés par les auteurs sont : porter un masque ou plusieurs couches de vêtements pour s'assurer que la peau n'est pas en contact avec le crabe, aller travailler à un poste ou un endroit différent dans l'usine et prendre une médication lors des pauses.

4 Cette tendance à gérer par soi-même sa maladie et ses douleurs pour parvenir à demeurer au travail ne semble pas uniquement présente chez les travailleurs-euses des usines de transformation du crabe de Terre-Neuve. Bien que cette tendance soit moins documentée au Québec, les travaux de Malo $(1988$; $1992 ; 1997)$ dans des usines de transformation du crabe aux Îles de la Madeleine laissent percevoir une situation semblable où les travailleurs ont recours à la médication pour gérer eux-mêmes leur maladie plutôt que de recevoir des indemnités de la CSST. D’ailleurs, Neis $(1994 ; 2001)$ avait soulevé un parallèle quant à la situation des travailleurs et travailleuses de Terre-Neuve et de ceux du Québec où l'on semblerait préférer gérer sa maladie par voie médicamenteuse pendant la saison plutôt que de s'absenter.

5 Ces résultats amènent à penser que les travailleurs-euses des usines de crabe pourraient également avoir recours à cette forme de gestion de la maladie pour faire face aux TMS. Les propos d'un médecin de la santé publique de la Côte-Nord (Québec) (Chrétien, 2006) soulignaient en ce sens la difficulté de traiter cette population en raison de sa quasiabsence en bureau de consultation médicale lors de la saison de travail. Certains 
travailleurs-euses consulteraient, mais ce n'est qu'en début de saison et ces derniers consultent pour demander des anti-inflammatoires et des injections de cortisone pour diminuer leurs douleurs afin d'être en mesure de faire leur saison de travail. De plus, cet intervenant du système de la santé déplorait la sous-déclaration des TMS à la CSST et le nombre très restreint d'études sur l'état de santé de cette population et insistait sur la nécessité d'actions au sein même de ces milieux de travail.

Par ailleurs, très peu d'études se sont attardées à la question de la santé au travail et du travail saisonnier (Neis, 1994 ; Schweder, 2008; Quinlan, 2010). Les quelques résultats laissent percevoir une réalité qui gagnerait à être mieux connue afin d'adapter ou encore de développer des mécanismes de prévention et de réparation qui tiennent compte des caractéristiques du travail saisonnier, ainsi que de la réalité à laquelle font face ces travailleurs-euses. De cette incertitude quant à la durée d'emploi découle une incertitude quant à l'éligibilité à l'assurance-emploi (Thériault, 2007). C'est dans ce contexte difficile que les travailleurs-euses développent des stratégies pour demeurer au travail.

\section{Objectifs}

$7 \quad$ Il apparait donc pertinent de documenter les stratégies que les travailleurs-euses d'usines de transformation du crabe mettent en œuvre pour gérer leur douleur et de se questionner sur les bénéfices et les préjudices potentiels liés à ces stratégies. L'un des objectifs de cette recherche en ergonomie a donc été de décrire la nature et les enjeux des stratégies développées par deux groupes de travailleuses, l'un dans une usine du Québec et l'autre dans une usine de Terre-Neuve.

Par ailleurs, bien que l'analyse des stratégies de personnes en activité de travail soit souvent au cœur des recherches en ergonomie, il existe très peu de cadres méthodologiques qui permettent de relever et de classer les stratégies mises en œuvre. Cet article vise principalement à présenter un cadre de référence pour le recueil et l'analyse des stratégies. Ce cadre de référence a été élaboré et testé empiriquement dans cette étude.

\subsection{Qu'est-ce qu'une stratégie ?}

9 D'abord faut-il savoir ce qu'on entend par une «stratégie ». Lorsque l'on consulte des travaux en ergonomie, particulièrement les travaux relevant de l'approche centrée sur l'activité de travail (Guérin et coll., 2006), il est très fréquent de rencontrer le terme « stratégie ». Qu'elles soient individuelles ou collectives, les stratégies peuvent avoir pour but de répondre aux exigences de production, de réduire les risques d'incidents, d'erreurs ou de débordements, mais également de protéger la santé et de réduire les risques d'accidents. Les conditions et moyens offerts par l'entreprise amènent la personne en activité à reconstruire ses tâches et à développer des modes opératoires et des stratégies dans un objectif d'équilibre entre ce qu'elle est (avec ses douleurs ressenties) et les attentes de production de l'entreprise (Vézina, 2001 ; Guérin et coll., 2006). En ce sens, une stratégie est élaborée pour permettre de tenir compte à la fois de sa propre variabilité et de la variabilité des conditions offertes par l'entreprise compte tenu de l'environnement externe de travail (Major, 2011), et ce, dans le but de maintenir un équilibre entre sa santé et sa production. 

de mieux analyser les situations de travail. "Stratégies de production", "stratégies de travail », "stratégies protectrices", "stratégies compensatoires", "stratégies de préservation de la santé " constituent quelques exemples couramment rencontrés (Authier, 1996; Gaudart, 1996; Bourgeois et coll., 2006 ; 2000 ; Cloutier et coll., 2005 ; Toupin, 2005 ; Denis et coll., 2007 ; Caroly, 2010 ; etc.). En dépit de la fréquente utilisation du terme, peu de travaux se sont arrêtés pour définir le sens qu'ils lui accordent . La plupart des travaux précisent à quelles fins les stratégies sont développées, mais demeurent imprécis et même muets quant à la définition qu'ils accordent au terme «stratégie ». Est-ce une action ou une intention? Qu'en est-il des inactions et des dénis, constituent-ils des stratégies? En d'autres termes, que considère $t$-on comme une stratégie? À notre connaissance, aucun cadre de référence des différents types de stratégies développées par les travailleurs n'est à la disposition des ergonomes et des chercheurs. Pourtant, un cadre de référence serait utile pour enrichir les échanges entre l'ergonome et la personne au travail.

11 En effet, plusieurs ont souligné la difficulté d'accéder à des dimensions du vécu de l'action qui ne sont pas immédiatement présentes à la conscience de la personne (Vermersch, $1989 ; 1991 ; 1994$; Clot et coll., 2000 ; Magnier et Werthe, 2001). Cette difficulté se pose à la fois pour l'intervieweur qui doit questionner ou observer les stratégies élaborées par un travailleur et pour l'interviewé qui doit expliciter ses actions et ses choix. Comme le souligne Vermersch (1991, p. 66), «très souvent l'intervieweur n'obtient pas de réponse ou des réponses à côté de la part du sujet». Un tel outil permettrait de guider l'intervieweur dans son processus de documentation des stratégies et ainsi orienter l'interviewé pour qu'il puisse rendre compte le plus finement possible de ce qu'il fait réellement et de comment il s'y est pris.

12 Selon le Centre National de Ressources Textuelles et Lexicales (CNRTL, 2010), une stratégie désigne un «ensemble d'actions coordonnées, d'opérations habiles, de manœuvres en vue d'atteindre un but précis ». Cette définition fait ressortir deux aspects intéressants à prendre en considération. D'une part, on comprend qu'une stratégie peut correspondre à des actions, des opérations et/ou des manœuvres que l'on considère coordonnées et habiles. D'autre part, la stratégie est développée « en vue d'atteindre un but précis ». Ce dernier aspect du concept de «stratégie » ressort clairement de la grande majorité des travaux en ergonomie ayant porté sur l'étude de stratégies. Dès 1972, Laville, Teiger et Duraffourg rapportaient les stratégies de travailleuses dans l'électronique qui modifiaient l'ordre des opérations dans leur cycle de travail « afin de gagner du temps ». Que ce soit les façons de faire des travailleurs expérimentés dans l'automobile pour se préserver de la fatigue et augmenter leur marge de manœuvre (Gaudart, 1996) ou faciliter la mémorisation des opérations (Vézina et coll., 2003) ; que ce soit les stratégies mises en œuvre par des téléphonistes à horaires très variables pour faciliter la conciliation travailfamille (Prévost et Messing, 2001) ; que ce soit les stratégies de travail des infirmières de nuit pour faire face à la baisse de vigilance nocturne (Toupin, 2005); que ce soit les stratégies des auxiliaires familiales concernant une meilleure connaissance des patients afin d'anticiper plus efficacement les risques (Cloutier et coll., 2005), la plupart des travaux font ressortir une partie importante de la définition de «stratégie ", soit celle d'être mise en œuvre « en vue d'atteindre un but précis ».

Chez certains auteurs, le terme «modalités de régulation » semble employé aux mêmes fins que le terme «stratégie » (Toupin, 2005; Gonzalez et Weill-Fassina, 2005; Caroly, 
2001). Chez d'autres auteurs (Chassaing, 2006; Ouellet et Vézina, 2008), les savoir-faire correspondent également à l'utilisation de stratégies, dans le sens d'opérations habiles et d'actions coordonnées, toujours selon l'atteinte d'un objectif.

Ces travaux mettent en évidence que par son activité le travailleur tente de s'adapter et de s'ajuster. Ainsi, par différents moyens relevant pour la plupart de l'activité de travail, tels que des modes opératoires, des savoir-faire, des façons d'utiliser les équipements de travail, des postures et des gestes, des gestions du temps de travail, des communications, des attitudes, des réélaborations de règles, des omissions à l'application de certaines règles, etc., ces travaux mettent en évidence l'ajustement, l'adaptation ou la régulation que met en place le travailleur pour rencontrer les exigences de production tout en tentant de préserver sa santé. La notion de stratégie est intimement liée au concept de régulation.

Outre ces stratégies qui se situent davantage au cœur même de l'activité de travail (modes opératoires, savoir-faire de prudence, etc.), Gonon (2003) propose un autre type de régulation qu'il désigne "régulations organisationnelles ». Il souligne que la régulation organisationnelle se situe sur le plan de la tâche prescrite et peut se traduire, notamment, par un changement de poste de travail au sein du service d'origine ou dans un autre service (ce qu'il appelle la « régulation réaffectation») ou peut également correspondre à un aménagement du poste au sein du service d'origine du travailleur tel que, par exemple, passer au travail de jour («régulation réaménagement») ou être un changement «total » de la tâche prescrite, c'est-à-dire par un changement de poste du travailleur à une autre profession («régulation reclassement»). Les régulations organisationnelles se traduisent par des reclassements, des réaffectations ou des réaménagements. Comme le font remarquer Gonzalez et Weill-Fassina (2005), la « régulation organisationnelle » comme l'appelle Gonon (2003) se situe à un autre niveau que la régulation sur le plan de l'activité de travail. Effectivement, la «régulation organisationnelle» (Gonon, 2003) déborde du cadre de celle qu'aborde la plupart des travaux communément retrouvés en ergonomie en ce sens que l'activité de travail n'est pas au cœur même des moyens par lesquels l'individu tente de retrouver ou reconstruire l'équilibre du système. Cette régulation dont parle Gonon (2003) concerne plutôt la gestion de l'emploi et des ressources humaines de l'entreprise pour tenter de répondre aux demandes du personnel ayant de la difficulté à faire face aux exigences de leur travail. Toutefois, selon notre point de vue, ce type de régulation pourrait aussi bien être élaboré par un travailleur, dans la mesure où il dispose de suffisamment de marge de manœuvre pour agir ainsi. Prenons l'exemple d'un travailleur sur une ligne de montage dont le poste de travail est situé tout près de la porte du congélateur et qui ressent une augmentation de ses douleurs en présence de courants d'air froid. Ce dernier pourrait demander à son superviseur d'aller travailler à un poste libre sur la chaîne de montage et qui n'est pas près du congélateur afin de préserver sa santé. Cette relocalisation ou "régulation réaffectation» comme la désigne Gonon (2003) peut être réalisée par l'entreprise mais, comme notre exemple l'illustre, également par le travailleur qui exprime une requête auprès d'autres personnes pour modifier sa situation de travail.

C'est également en ce sens qu'un travailleur pourrait élaborer des stratégies en dehors du cadre du travail et de l'activité de travail, mais qui seraient développées dans le même objectif que celles se situant dans l'activité de travail, c'est-à-dire en visant à retrouver ou à reconstruire un équilibre entre les ressources de l'individu, notamment cognitives, physiques et sociales, et les caractéristiques de l'environnement de travail. Tout comme 
les travaux de Curie et Hajjar (1987) le font ressortir, les sphères « vie de travail » et « vie hors travail» constituent des sphères autonomes, mais qui sont également interdépendantes dans le sens où une modification de l'une entraîne « la mobilisation de processus de régulation du système global d'activités » (Curie et Hajjar, 1987, p. 52).

À titre d'exemple, une travailleuse pourrait développer comme stratégie de diminuer sa charge de travail à la maison en se faisant aider par son conjoint afin d'être en mesure de réaliser son travail à l'usine ou encore frotter son épaule avec une crème le soir après le travail pour diminuer la douleur afin d'être capable de reprendre le travail le lendemain. En ce sens, ces stratégies qui relèvent davantage du plan "personnel et médical» rejoignent la stratégie de " prendre des médicaments pour gérer sa maladie » développée par les travailleuses d'usines de transformation du crabe et qui ressort de plusieurs des travaux sur l'asthme professionnel du crabe (Howse et coll., 2006 ; Gautrin et coll., 2010 ; etc.).

18 Par conséquent, cette étude s'est intéressée non seulement aux stratégies relevant de l'activité de travail tel qu'il est classique de retrouver en ergonomie, mais également aux stratégies débordant du cœur même de l'activité de travail, soit celles reliées à des requêtes formulées auprès d'autres personnes (ressources humaines, maintenance, etc.) pour modifier leur situation de travail et celles sur le plan personnel et médical.

Par ailleurs, compte tenu de l'objectif de cette étude, soit de documenter les stratégies développées par des travailleuses, et basé sur la revue de différents travaux en ergonomie ayant porté sur l'étude des stratégies, la définition suivante du terme "stratégie » est proposée : «Les stratégies ou modalités de régulation sont des comportements, des savoirfaire, des attitudes que la personne développe pour parvenir à maintenir l'équilibre entre ce qu'elle est et son environnement ».

Tout comme il ressort des différents travaux en ergonomie, ces stratégies peuvent être élaborées par les travailleurs pour rencontrer les exigences de production, augmenter leur propre niveau de satisfaction par rapport à la qualité de leur travail, etc. Compte tenu de la situation particulière des travailleuses de l'industrie de la transformation du crabe mise en évidence par les travaux sur l'asthme professionnel du crabe, cette étude s'intéressera aux stratégies élaborées par ces dernières pour gérer leur douleur.

\section{Méthodologie}

21 La méthodologie utilisée dans cette recherche émane de la combinaison de trois modèles. Il s'agit en tout premier lieu d'une étude ergonomique réalisée selon l'approche centrée sur l'analyse de l'activité de travail (Guérin et coll., 2006; Daniellou, 2005). Une des caractéristiques de cette approche est d'apporter une compréhension des difficultés rencontrées par les travailleurs-euses lors de la réalisation de leur activité de travail et des ajustements mis en œuvre par ceux-ci pour faire face à la variabilité de leur cadre de travail et de leur propre variabilité. L'analyse des modes opératoires et des stratégies développés par les travailleurs-euses pour faire face à la variabilité ainsi qu'aux difficultés rencontrés est au cœur de cette approche. Cette fine compréhension de l'activité de travail mène à l'identification et l'explication de déterminants contribuant aux difficultés rencontrées par les travailleurs-euses et aux effets sur la santé et la production. Le lien très étroit entre la recherche et l'intervention permet notamment de valider les modèles théoriques développés (Daniellou, 2005). 

Burgess (1986) et Sieber (1982). Une des particularités de cette approche est l'immersion $\mathrm{du}$ chercheur dans le milieu sur une période suffisamment longue pour permettre une compréhension détaillée de la situation à l'étude. De plus, pour des études se déroulant dans les régions rurales, cette approche semble particulièrement indiquée. Se basant sur les résultats de différentes études portant sur les régions éloignées, Burgess (1986) constate qu'une immersion du chercheur dans ces milieux permet à ce dernier d'en être imprégné, ce qui apporte une richesse et une profondeur aux résultats.

d'une étude de cas multiples à niveaux d'analyse imbriqués (Yin, 2003). L'unité principale d'analyse, en d'autres mots le " cas » étudié, est définie par une travailleuse œuvrant dans une usine de transformation du crabe. Yin (2003), l'un des auteurs les plus cités concernant les études de cas (Dopson, 2003), définit l'étude de cas comme une méthode d'investigation empirique de phénomènes contemporains dans leur contexte naturel. Toujours selon Yin (2003), l'étude de cas se distingue par la contribution qu'elle apporte à la compréhension de systèmes complexes, tels les systèmes sociaux, les systèmes humains. L'étude de cas permet au chercheur de comparer des phénomènes empiriques à des phénomènes prédits ou encore induire un modèle théorique à partir de l'étude d'un ou de plusieurs cas observés dans leur contexte naturel. L'étude de cas relève des approches de recherche synthétiques (théorique et interprétative) qui ont pour but l'analyse de phénomènes complexes du monde réel (Contandriopoulos, Champagne, Potvin, Denis et Boyle, 1990 ; Collerette, 2004). Contandriopoulos et coll. (1990) perçoivent en l'étude de cas un devis de recherche qui permet l'explicitation et la généralisation d'une théorie. D'après ces auteurs (Contandriopoulos et coll., 1990), la puissance explicative découle de la profondeur de l'analyse du cas et non du nombre des unités d'analyse étudiées. Eisenhardt (1989) abonde également en ce sens en soulignant que la représentativité du cas est secondaire et que c'est la qualité même du cas qui devient le souci principal du chercheur. Chacun des cas représente donc un objet unique de recherche. De plus, d'après Yin (2003), il est préférable de recourir à l'étude de cas lorsqu'il s'agit de répondre à des problèmes de liens opératoires qui doivent être étudiés pendant un certain temps, plutôt que de faire part de fréquences ou d'incidences de phénomènes. L'étude de cas permet d'enrichir et de généraliser des théories (généralisation analytique) plutôt que d'énumérer des fréquences et des statistiques (généralisation statistique) (Yin, 2003). Le recours à plusieurs cas (étude de cas multiples) permettra de faire ressortir des convergences entre plusieurs cas, tout en contribuant à l'analyse des particularités de chacun d'eux. En ce sens, l'étude de cas multiples nous apparaît particulièrement indiquée pour parvenir à induire un cadre de référence pour l'étude des stratégies des travailleurs-euses. De plus, sa grande flexibilité, qui facilite l'utilisation de multiples sources d'informations (Yin, 2003), permettra de prendre en compte les caractéristiques des autres modèles méthodologiques auxquels cette étude a recours.

\subsection{Le choix des sujets}

Seize travailleuses de l'industrie de la transformation du crabe ont été sélectionnées. Le choix des travailleuses a été effectué dans une logique de réplication théorique où des cas 
globalement semblables, mais contrastant sur une (ou des) dimension(s) prédéterminée (s), sont volontairement choisis afin de produire des résultats contrastants (Yin, 2003).

Tous les cas suivis étaient de sexe féminin. Ce choix était basé sur les résultats de différentes études mettant en évidence que les TMS représentent un problème majeur chez les travailleuses d'usines de transformation des produits de la mer (Messing et Reveret, 1983 ; Ohlsson et coll., 1994 ; Norander et coll., 1999 ; Solberg et coll., 2005). Par ailleurs, les travailleuses suivies devaient vivre des épisodes de douleur au travail. Ce critère de sélection des cas était en cohérence avec la question et les objectifs de recherche. En effet, rappelons que nous nous intéressons dans cette étude aux stratégies développées dans le but de gérer la douleur.

De plus, comme les auteurs s'intéressaient aussi aux stratégies personnelles développées hors travail pour parvenir à se maintenir au travail, il était tout indiqué de viser une population de femmes, lesquelles sont reconnues comme portant le plus souvent la responsabilité de s'occuper de la maisonnée et des tâches ménagères.

Sur les 16 travailleuses suivies, huit d'entre elles provenaient d'une usine située au Québec (Côte-Nord) et les huit autres, d'une usine de Terre-Neuve. Outre cette dimension de contraste, le choix des travailleuses a également été effectué en fonction du (ou des) poste(s) de travail occupé(s). Ce critère constituait un élément de contraste (réplication théorique) quant à l'élaboration de stratégies par les travailleuses en permettant de couvrir un large éventail de situations de travail possibles (empaquetage, pesage, emballage, transformation de la chair de crabe, etc.) (voir en annexe). Les sujets potentiels ont été choisis à l'aide d'une personne responsable des ressources humaines au sein de chaque usine à partir de ces critères. La participation des sujets était sur une base volontaire et la chercheuse a rencontré chacun d'entre eux individuellement pour leur présenter le projet et s'assurer de leur intérêt à prendre part à l'étude.

\subsection{Caractéristiques de la population étudiée}

28 La moyenne d'âge et d'ancienneté pour les huit travailleuses sur la Côte-Nord est de 46 ans $(+/-7,2)$ et 12 années $(+/-3,6)$ et pour celles de Terre-Neuve, 47 ans $(+/-5,2)$ et 23 années $(+/-5,7)$ (données en date de 2005). D'une année à l'autre, ces travailleuses reviennent à l'usine pour la saison de crabe puisque aucune autre possibilité d'emploi n'existe dans ces régions éloignées. Seules deux travailleuses ont déjà occupé un autre emploi pendant quelques semaines lors de la période hors saison depuis qu'elles travaillent à l'usine.

La situation familiale des travailleuses était très variable de l'une à l'autre. Certaines travailleuses avaient des enfants en bas âge contrairement à d'autres dont les enfants n'habitaient plus au domicile familial ou, s'ils y habitaient, étaient autonomes. Des différences entre les travailleuses suivies étaient également présentes quant au statut matrimonial.

30 Ces différences ont permis d'aborder un large éventail de situations possibles. Tout comme le souligne Stake (1995), dans la sélection du cas, la possibilité d'apprendre est primordiale. La compréhension des aspects uniques de chaque cas dans une étude de cas multiples est essentielle pour élargir la compréhension d'un phénomène humain.

31 Une grande variation était également présente entre les travailleuses concernant le nombre et les sites corporels de douleur rapportés. Cependant, la plupart (14/16) des 
travailleuses ont mentionné des douleurs aux épaules, et ce, pour la durée complète de la saison. Sur l'ensemble des travailleuses suivies, toutes celles de l'usine de Terre-Neuve ressentaient des douleurs de façon chronique, c'est-à-dire durant la saison de travail et la période hors saison. Pour les travailleuses de l'usine de la Côte-Nord, la moitié de celles suivies (4/8) ont rapporté des douleurs présentes durant la saison et la période hors saison. Les autres travailleuses de l'usine de la Côte-Nord (4/8) ont rapporté des douleurs qui pourraient être caractérisées de "saisonnières et journalières", c'est-à-dire des douleurs présentes durant la saison de travail qui apparaissent au cours de la journée de travail, et ce, à tous les jours durant la saison de travail, mais ne sont pas nécessairement présentes durant la période hors saison. Cette situation accentuait la nécessité pour les travailleuses de développer des stratégies pour rester au travail.

\subsection{Caractéristiques des contextes et des usines}

Les deux usines étaient situées au sein de localités comptant entre 2500 et 3000 habitants. Malgré la présence d'une diversité économique quelque peu différente, les possibilités d'emplois demeurent limitées au sein des deux localités. Au cours de la saison 2005, l'usine de Terre-Neuve comptait 314 travailleurs affectés à la production et l'usine de la Côte-Nord, 130. Le procédé de transformation du crabe employé aux deux usines est similaire, mais on trouve une plus grande parcellisation des tâches à l'usine de TerreNeuve où les équipements sont plus modernes. Une description détaillée de l'activité de travail et des conditions du travail au sein de ces deux usines de transformation du crabe est présentée dans Major (thèse de doctorat, 2011).

\subsection{Triangulation des méthodes de collecte des données}

Plusieurs méthodes de collecte des données ont été utilisées dans une logique de triangulation des méthodes, et ce, conformément aux recommandations pour accroître la validité des résultats dans les études de cas (Yin, 2003). Cette façon de procéder est également propre à la démarche ergonomique (Guérin et coll., 2006) et en fait l'une de ses forces. Des entretiens, des schémas corporels, des rencontres d'autoconfrontation, ainsi que des observations de l'activité et de l'organisation du travail constituent les principales sources de données utilisées pour documenter les stratégies élaborées par les travailleuses pour gérer leur douleur. Soulignons que ces méthodes combinées à l'analyse de documents avaient également pour but de documenter les caractéristiques du travail saisonnier et de faire un suivi de l'état de santé musculo-squelettique de ces travailleuses. Ces objectifs seront abordés dans des articles distincts. Ajoutons que tout au long de la collecte des données, un journal de bord a été tenu par la chercheuse et rassemble l'ensemble des notes de terrain prises au cours des deux années.

Les propos qui suivent présenteront d'abord le déroulement de l'étude pour permettre de visualiser le calendrier de la collecte des données, ainsi que de comprendre l'articulation entre chacune des méthodes utilisées. Par la suite, les spécificités de chacune de ces méthodes seront décrites. Au sein des figures présentées, un numéro est attribué à chaque méthode et ce numéro est repris dans le texte lors de la présentation des méthodes. 


\subsection{Déroulement de l'étude}

Le suivi des travailleuses a été réalisé pendant deux saisons consécutives, soit 2005 et 2006. La figure 1 montre le déroulement de la collecte des données au sein des deux usines pour les deux saisons de suivi. Les parties A et B de la figure 1 présentent les méthodes de cueillette et d'analyse des données pour l'usine de la Côte-Nord et de TerreNeuve, respectivement, pour la première saison (2005). Pour chacune des usines, les dates de début, de fin et de retour au travail lors de la saison suivante y sont présentées. La présence de la chercheuse à chacune des usines y est représentée par la zone hachurée. Cette présence fut continue, c'est-à-dire qu'à chaque journée où il y avait de la production, la chercheuse était à l'usine, et ce, peu importe la journée de la semaine pour être en mesure de suivre la production et de comprendre la dynamique du travail saisonnier. Aux points A et B de la figure 1, les diverses méthodes de cueillette et d'analyse des données sont indiquées ainsi que le moment où celles-ci ont été utilisées (saison, hors saison, lors de la présence de la chercheuse à l'usine, etc.). La première année de collecte et d'analyse des données (figure $1 \mathrm{~A}$ et $\mathrm{B}$ ) a mené à une première identification des stratégies développées par l'ensemble des travailleuses suivies (figure 1 C). Pour la seconde année, les méthodes de recueil et d'analyse des données sont présentées à la figure $1 \mathrm{D}$ et $\mathrm{E}$ pour l'usine de la Côte-Nord et Terre-Neuve, respectivement. Le recueil des données de la deuxième saison a profité du fait que la chercheuse avait déjà connaissance d'un ensemble de stratégies. Ainsi, la grille développée en $\mathrm{C}$ a servi à approfondir le questionnement sur les stratégies auprès des travailleuses au cours de la deuxième saison. L'ensemble des étapes a mené à l'élaboration d'un cadre de référence pour l'analyse des stratégies (figure 1D).

Figure 1. Déroulement de la collecte des données au sein des deux usines pour les deux années de suivi

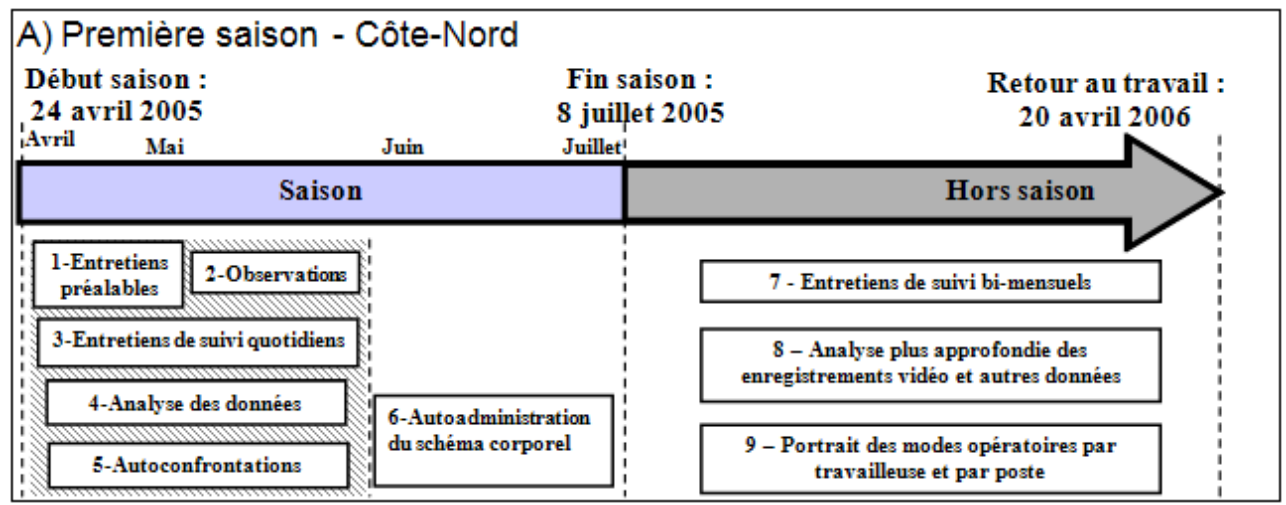




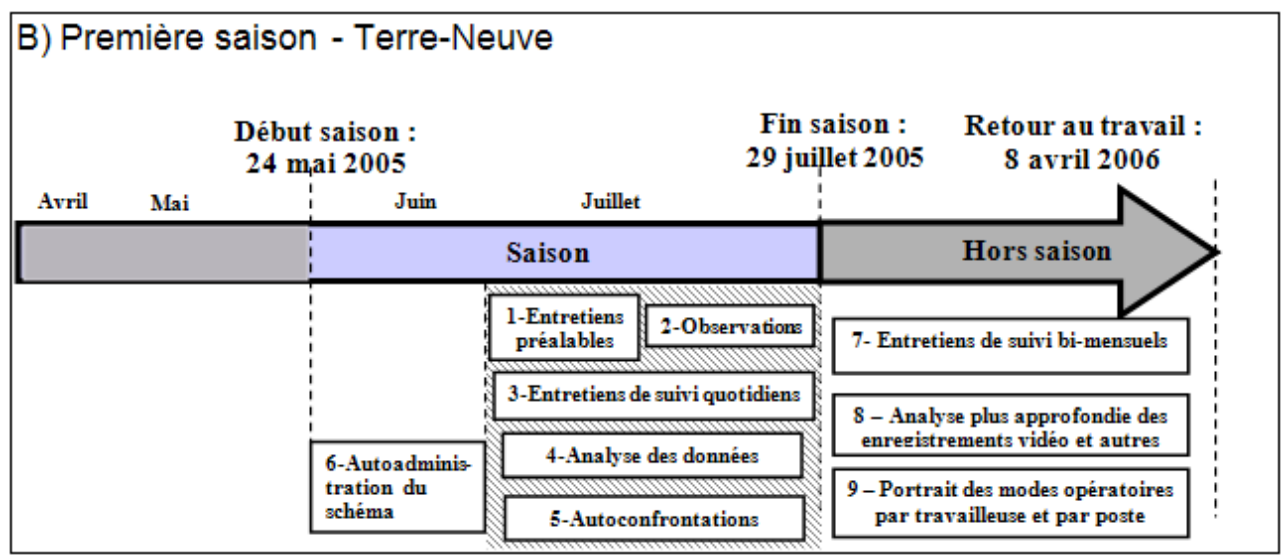

\section{C) Élaboration d'une première grille des stratégies sur la base des portraits} des modes opératoires des 16 travailleuses (Côte-Nord et Terre-Neuve)

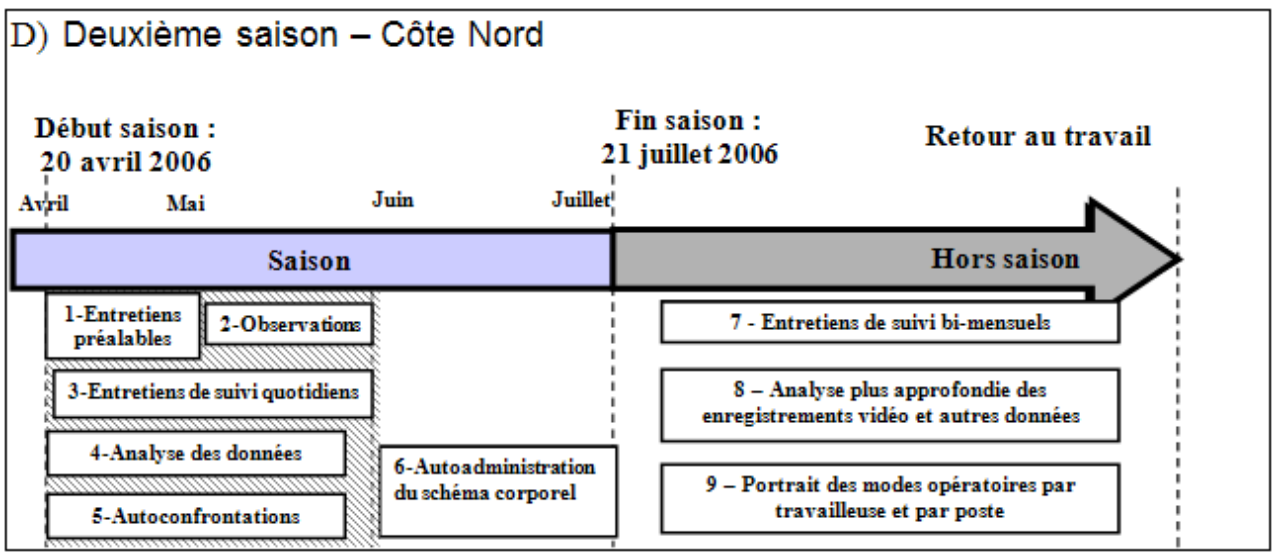

Les spécificités de chacune de ces méthodes et étapes sont présentées dans les propos qui suivent. Un suivi au cours de la période hors saison a également été effectué pour les périodes 2005 et 2006, mais les données collectées lors de cette période ainsi que pour celles de l'étude pilote ne font pas l'objet du présent article.

\subsection{Cueillette et analyse des données : un processus itératif}

Dans les propos qui suivent, un aperçu des méthodes utilisées sera présenté. D’entrée de jeu, soulignons que des informations nouvelles recueillies au cours de la collecte des données ont entraîné certaines modifications en cours de route. Cette flexibilité correspond à l'une des forces des études de cas, soit celle de pouvoir s'adapter aux réalités rencontrées sur le terrain (Yin, 2003). Comme on l'a vu, des premières analyses ont été réalisées en cours de projet. D'une part, ces analyses ont permis la validation des premiers résultats et, d'autre part, cette façon de procéder assurait une profondeur et une richesse des résultats. Les données obtenues par les premières analyses constituaient non seulement des éléments de discussion lors de la rencontre d'autoconfrontation avec la travailleuse, mais permettaient également de documenter si ces stratégies étaient mises en œuvre par cette même travailleuse sur d'autres postes et si les autres travailleuses avaient également recours à cette stratégie. Cette démarche avait pour effet 
de minimiser les impacts sur les résultats d'une travailleuse qui aurait des difficultés à verbaliser ses façons de faire.

\subsubsection{Entretiens individuels préalables} Vézina, Ouellet et Major, 2009) ont eu lieu avec chacun des sujets. Un canevas d'entretien avait été préparé au préalable et les questions à aborder étaient dérivées du modèle conceptuel proposé par Vézina (2001) et davantage développé dans cette recherche (thèse de doctorat, à paraître). Les versions en français et en anglais des canevas d'entrevue avaient été validées auprès de collaborateurs. Pour la seconde année, le même canevas a été administré, mais servait plutôt de guide pour relever tout changement comparativement à la première année.

\subsubsection{Observations}

\section{a. Observations de l'activité et verbalisations}

Un premier travail d'identification des stratégies développées par les travailleuses pour gérer leur douleur a été réalisé par le biais d'observations de l'activité de travail de chacune des travailleuses en situation réelle. Pour ce faire, la chercheuse a d'abord relevé (méthode crayon/papier) les mouvements, les façons de faire, les postures adoptées, les outils utilisés, les entraides avec une collègue de travail, la présence de communications/ d'échanges avec d'autres et tout autre élément qui était observable en temps réel (Guérin et coll., 2006). Les caractéristiques de l'aménagement du poste de travail étaient également décrites. Par la suite, les observations ont été réalisées davantage dans une perspective d'approfondir la compréhension du travail où une attention particulière a été portée aux raisons qui pouvaient sous-tendre chaque observable et à tout signe qui pouvait être relié à une manifestation de douleur exprimée par la travailleuse. Par exemple, la chercheuse se questionnait sur le pourquoi une travailleuse se positionnait de telle façon par rapport à ses paniers, sur le pourquoi réaliser telle opération avant telle autre, etc. Cette façon d'observer permettait de relever les pistes à approfondir et à valider lors des rencontres d'autoconfrontation. La méthode de l'observation réalisée pourrait être qualifiée d'« observation participante » au sens où il y a eu «immersion intensive et intervention minimale de la chercheuse » (Mongeau, 2008, p. 96).

40 À quelques reprises lors des observations, des verbalisations informelles ont eu lieu afin d'approfondir certains aspects. Ces échanges sporadiques pouvaient provenir autant de la travailleuse (par exemple, expliquer ses façons de faire, prendre conscience d'un ajustement dans la façon de faire en raison d'une douleur, etc.) que de la chercheuse (par exemple, questionner sur le pourquoi d'une façon de faire, etc.). La chercheuse s'est assurée que ces échanges n'influençaient pas le cours de l'activité de travail et de la production.

\section{b. Observations de l'organisation du travail et des événements liés à la production}

41 L'observatrice a également été attentive aux événements qui se sont produits dans les départements et les a notés de façon chronologique pendant plusieurs journées d'observations (papier/crayon). Ces événements concernaient, par exemple, l'ajout ou le retrait d'une personne sur la chaîne de production, le changement de type de produit, le

Perspectives interdisciplinaires sur le travail et la santé, 13-2 | 2011 
bris d'une machine et les ajustements conséquents dans le personnel, la rotation des postes, l'arrivée d'un apprenti sur la chaîne, etc. Ces observations ont servi à réaliser un portrait au quotidien de l'organisation du travail, de la production, de leur variabilité, ainsi que de tout incident et des ajustements apportés. Les démarches effectuées par la travailleuse auprès des superviseurs et de ses collègues de travail pour apporter des changements ayant une influence sur sa douleur ont également été notées (par exemple, remplacement lors d'une pause). Au total, des observations en situation réelle ont été réalisées sur près de 19 semaines (Côte-Nord : $2005: 4$ semaines, 2006: 4 semaines ; TerreNeuve : $2004: 3$ semaines, $2005: 4$ semaines, $2006: 4$ semaines).

\subsubsection{Entretiens de suivi quotidiens}

Ces entrevues avaient pour but de questionner la travailleuse quant aux inconforts rapportés sur le schéma corporel et aux stratégies développées pour les diminuer ou les contrôler. Pour chacune des 33 régions corporelles (Vézina, Ouellet et Major, 2009 ; Major et Vézina, 2007), les travailleuses devaient indiquer le niveau d'inconfort (1 à 5) qu'elles ressentaient.

Ces courts entretiens ont été réalisés à chaque début de journée de travail, ainsi qu'à la fin (durée : 5 à 15 minutes). Le but principal de ceux-ci était de questionner la travailleuse quant aux inconforts (régions corporelles et degrés d'inconforts rapportés) et aux stratégies développées pour diminuer ou contrôler la douleur lorsque celle-ci avait noté un degré d'inconfort plus élevé que 1 sur son schéma corporel. Dans la mesure du possible, la chercheuse tentait de remplir le schéma corporel avec les travailleuses. Mais, si pour diverses raisons, il était difficile de réaliser l'entretien, les travailleuses procédaient de façon autoadministrée. Toutefois, précisons que la chercheuse s'est assurée de réaliser ces entretiens pendant au moins une semaine en début de saison avec chacune des travailleuses pour avoir la certitude que les travailleuses comprenaient le schéma corporel et indiquaient les informations appropriées quant aux stratégies développées sur leur schéma corporel lorsque besoin était.

Une étude pilote auprès des travailleuses de l'usine de Terre-Neuve avait d'abord été réalisée au cours de la saison de travail de 2004 pendant quatre semaines. Cette étude pilote a permis de valider le schéma corporel et d'apporter les précisions nécessaires aux travailleuses. Cette administration du schéma corporel au cours de la saison 2004 a permis aux travailleuses de Terre-Neuve de débuter la saison 2005 en procédant de façon autoadministrée pendant que la chercheuse commençait la saison sur la Côte-Nord (cf. figure 1).

\subsubsection{Une analyse détaillée des enregistrements vidéo}

45 L'analyse détaillée des enregistrements vidéo consistait à caractériser et décrire de manière élaborée chacune des façons de faire relevées. À titre d'exemple, pour l'opération "saisir la section de crabe sur le convoyeur ", des composantes telles que : la région du crabe saisie (par la patte, l'épaule, etc.) ; l'endroit sur le convoyeur où la section de crabe était saisie par rapport à la position de la travailleuse (devant soi, derrière soi, etc.); l'amplitude des mouvements articulaires (ROM) de l'épaule, du cou et du tronc (ex.: abduction de l'épaule de plus de 45 degrés, etc.) ; l'emplacement des paniers de crabe par rapport au corps de la travailleuse (devant elle, collé sur son ventre, etc.) ; la disposition des paniers de crabe (devant la travailleuse dans le sens de la longueur, sens de la largeur, 
etc.); l'omission et/ou l'ajout de certaines opérations; la répétition de certaines opérations (ex. : changer le crabe de main à plusieurs reprises); etc. Pour chacune des travailleuses suivies, un tableau a été élaboré rapportant, pour chacun des observables préalablement identifiés, les stratégies relevées et incorporant également les informations sur les stratégies recueillies lors des étapes précédentes.

\subsubsection{Rencontres d'autoconfrontation} la douleur au cou et aux membres supérieurs sur la vie quotidienne (IDVQ) (Stock et coll., 2003). Pour chacune des questions, un échange était engagé par la chercheuse pour tenter de mettre en lumière les raisons sous-jacentes à l'indice de limitation fonctionnelle donné par la travailleuse et de découvrir de quelle(s) façon(s) cette dernière parvenait à réaliser 
l'activité décrite en dépit de ses limitations fonctionnelles. Par exemple, pour la question : "Quel degré de difficulté avez-vous ou auriez-vous maintenant avec les activités ou les situations suivantes, à cause de votre problème : pour vous laver le dessus de la tête ou laver, sécher ou arranger vos cheveux?» (section A, question 2, page 1), la travailleuse évaluait d'abord son degré de difficulté à réaliser cette activité. Par la suite, la chercheuse tentait de cerner, par diverses questions, la façon dont la travailleuse y parvenait (par elle-même mais en réalisant cette activité d'une façon particulière, comme se sécher les cheveux la tête en bas pour ne pas avoir mal à l'épaule en tenant le séchoir ou encore grâce à l'aide de quelqu'un d'autre, comme sa fille qui lui sèche les cheveux, etc.).

\subsubsection{Autoadministration du schéma corporel}

50 Tout au long de la saison de travail, les travailleuses devaient compléter un schéma corporel. Il s'agit du même schéma corporel que celui présenté au point 3 (aspect 3 de la méthodologie, présenté ci haut) et les travailleuses le remplissaient de façon autoadministrée (deux fois par jour: début et fin de chaque journée de travail). Sur ce schéma corporel, un espace était également réservé pour que la travailleuse y note les stratégies développées, les médicaments (prescrits et non prescrits) consommés au cours de la journée et tout autre commentaire.

\subsubsection{Entretiens de suivi bi-mensuels}

51 Ces entretiens semi-dirigés ont permis de suivre l'évolution de chacune des travailleuses lors de la période hors saison. Au cours de ces entretiens, le schéma corporel a été complété et la travailleuse décrivait ses stratégies sur le plan personnel et médical. Les activités de la personne ont également été prises en note. Notamment, si cette dernière occupait un emploi, ses activités, ses passe-temps, etc. Ces entretiens, d'une durée d'environ 30 à 45 minutes, ont été réalisés au cours d'un entretien téléphonique à chaque deux semaines à partir du moment où la travailleuse terminait sa saison de travail. Les résultats de ces entretiens ne sont pas présentés dans cet article.

\subsubsection{Une analyse plus complète des enregistrements vidéo et autres données}

Ces analyses, qui se sont déroulées pendant la période hors saison, avaient pour but d'apporter un portrait plus complet des stratégies que celui obtenu à la suite des analyses effectuées par la chercheuse au cours de la saison de travail au moment où elle était à l'usine et devait préparer les rencontres d'autoconfrontation (aspect 4 de la méthodologie, présenté ci-haut). Lors de sa présence sur le terrain, compte tenu de la réalité du travail saisonnier (horaire irrégulier, longues heures de travail, etc.), il était difficile pour la chercheuse de visionner plusieurs enregistrements vidéo d'une même travailleuse, et ce, sur les différents postes que cette dernière avait occupés. Les analyses réalisées durant la période hors saison avaient donc le même but, soit caractériser et décrire chacune des façons de faire relevées, mais ces nouvelles analyses des enregistrements vidéo ont permis à la chercheuse de s'assurer de visionner au moins quatre enregistrements vidéo (durée de 20 à 30 minutes chacun) de la travailleuse à son poste assigné et, dans la mesure du possible, au moins deux enregistrements vidéo aux autres postes occupés ont été effectués. Pour les séquences vidéo au poste assigné, la chercheuse s'est assurée d'avoir au moins un enregistrement au début, en après-midi, en fin de journée de travail et un autre enregistrement lorsque les conditions s'y prêtaient. 
Ces analyses ont donné la possibilité de détailler davantage les différentes façons de faire d'une même travailleuse (intra-cas) et entre les travailleuses (inter-cas).

De plus, lors de cette période hors saison, les entretiens préalables et les rencontres d'autoconfrontation ont été transcrits et lus pour y repérer des stratégies élaborées par les travailleuses.

Avec l'ensemble des données, nous avons pu faire un portrait des modes opératoires par travailleuse et par poste (aspect 9, figure 1).

\section{7 Élaboration d'un cadre de référence pour l'étude des stratégies ( $F$ dans figure 1 )}

Les entretiens préalables ainsi que les rencontres d'autoconfrontation ont été enregistrés, transcrits et ont fait l'objet d'une analyse qualitative de contenu, réalisée dans une logique de découverte et de construction du sens (Mucchielli, 2006 ; Huberman et Miles, 2003). Les diverses étapes des analyses des données sont présentées à la figure 2. Ces analyses ont permis d'élaborer une première version du cadre de référence pour l'étude des stratégies avec les données de la Côte-Nord et de tester empiriquement cet outil avec l'usine de Terre-Neuve et de constituer des matrices conceptuelles hiérarchiques. Dans un deuxième temps, les analyses réalisées avaient pour but de décrire la nature et les enjeux des stratégies développées par les travailleuses.

Figure 2. Étapes de l'analyse des données

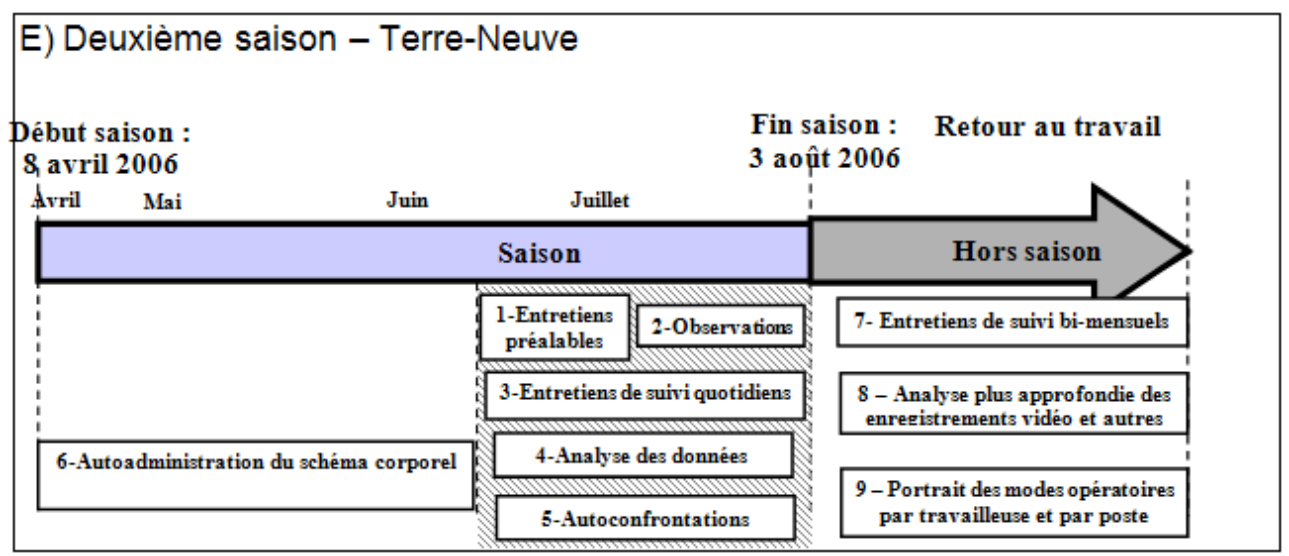

\subsubsection{Catégories émergentes et définitions : élaboration du cadre de référence} (figure 2, boîtes 1-2-3)

Dans un premier temps, les verbatim ont été relus pour y repérer les stratégies. Rappelons ce que l'on entend par stratégie : «Les stratégies ou modalités de régulation sont des comportements, des savoir-faire, des attitudes que la personne élabore pour parvenir à maintenir l'équilibre entre ce qu'elle est et son environnement ». Ces dernières ont été colligées dans une banque de données sous la forme de deux fichiers informatiques au format Excel ${ }^{\mathrm{TM}}$, l'un regroupant toutes les stratégies relevées auprès de l'ensemble des travailleuses suivies de l'usine de la Côte-Nord et l'autre pour celles des travailleuses de l'usine de Terre-Neuve. À l'intérieur de ce fichier, les stratégies ont été classées selon les trois sous-unités d'analyse : 1) activité de travail, 2) requêtes auprès d'autres personnes 
(superviseurs-contremaîtres, ressources humaines, direction, maintenance) pour modifier leur situation de travail et 3) médical et personnel.

En nous basant sur l'ensemble des stratégies développées par les travailleuses de l'usine de la Côte-Nord, des catégories ont été créées à partir d'une démarche inductive qui regroupait des éléments apparentés, ce que Miles et Huberman (2003) nomment « catégoriser » et «subdiviser les données ». Par la suite, une définition a été élaborée pour chaque catégorie obtenue à partir des ensembles conceptuels constitués par les données de stratégies regroupées. Cette façon de procéder assurait que les définitions étaient ancrées empiriquement dans les données recueillies sur le terrain. Ces étapes ont mené à une première version du cadre de référence.

\subsubsection{Tester empiriquement le cadre de référence (figure 2, boîtes 4-5)}

58 Ces étapes ont d'abord été réalisées avec les données de chacune des travailleuses de l'usine de la Côte-Nord et ont, par la suite, été répétées avec les données de chacune des travailleuses de l'usine de Terre-Neuve. Il s'agit d'une double validation, en ce sens que l'une est rattachée au devis de recherche utilisé «étude de cas multiples " et l'autre découle de la logique de réplication théorique de cette étude (deux usines, différents postes de travail au sein d'une même usine) (Yin, 2003). D’une part, ces étapes ont permis de vérifier si la catégorisation obtenue par les stratégies des travailleuses de l'usine de la Côte-Nord rencontrait également celle obtenue par les stratégies des travailleuses de l'usine de Terre-Neuve. D'autre part, cette étape de validation a permis de vérifier que les définitions élaborées à partir des stratégies des travailleuses de la Côte-Nord prenaient également en compte les stratégies obtenues des travailleuses de l'usine de Terre-Neuve pour chacune des catégories. Les définitions ont pu ainsi être raffinées. Ces étapes ont conduit à l'élaboration d'un cadre de référence (catégorisation des stratégies et définition de chaque catégorie) qui rend compte de toutes les données obtenues et qui est ancré empiriquement dans les données recueillies sur le terrain. Finalement, une étape de confirmation visant à confirmer ou contredire les interprétations précédentes a été assurée par le recours à une tierce personne (Yin, 2003). Les stratégies ont été discutées, une à une, avec la directrice de recherche afin de valider la stratégie : 1) est-ce bien une stratégie (en accord avec la définition du terme «stratégie » préalablement établie) ; 2) est-ce bien une stratégie développée pour gérer la douleur ; 3) est-ce que les catégories et les définitions élaborées, ainsi que le classement des stratégies (données brutes) dans les catégories sont adéquats?

59 À l'issue du processus de codage, les stratégies contenues dans les deux fichiers informatiques au format Excel ${ }^{\mathrm{TM}}$ (usine de la Côte-Nord et usine de Terre-Neuve) ont été insérées dans un fichier les regroupant par classe et par catégorie pour chaque travailleuse et à chacun des postes occupés par celle-ci pour obtenir des matrices conceptuelles hiérarchiques (Miles et Huberman, 2003).

\subsubsection{Codage thématique : nature des stratégies développées (figure 2, boîte 6 )}

Les analyses suivantes avaient pour but de découvrir à l'intérieur des catégories de stratégies obtenues les "patterns ", c'est-à-dire les thèmes communs qui ressortent des stratégies au sein de chacune des catégories. Cette étape correspond à ce que Miles et Huberman (2003) nomment "repérer les patterns » et "subsumer le particulier sous le général ». Les thèmes obtenus correspondent à la nature et aux enjeux des stratégies 
développées par les travailleuses pour gérer leur douleur (par exemple, «compromis coûteux pour la santé»). Pour s'assurer de la justesse des thèmes obtenus et de leur interprétation, les verbatim contenant les stratégies découvertes lors des premières analyses ont été relus. Une consultation des notes prises par la chercheuse lors de la collecte des données (journal de bord) a également été effectuée. Ces relectures ont permis de s'assurer de la contextualisation des résultats et de sélectionner des verbatim tirés des verbalisations des travailleuses pour illustrer les résultats obtenus.

\subsection{Considérations déontologiques}

61 Le protocole de recherche a reçu l'approbation du comité institutionnel d'éthique de la recherche avec des êtres humains de l'UQAM et de Memorial University.

\section{Résultats}

62 Tout d'abord, afin de bien situer et comprendre les résultats, rappelons que le processus de régulation d'une personne au travail vise à maintenir un équilibre entre les caractéristiques et les ressources personnelles de l'individu, notamment cognitives, physiques et sociales, et les caractéristiques de son cadre de travail tel que défini dans StVincent et coll. (2011). Cette recherche a la particularité d'étendre le concept de régulation à l'activité déployée hors travail (tâches ménagères, loisirs, soins personnels, etc.) par la travailleuse. Ce processus de régulation se manifeste par des stratégies ou des modalités de régulation qui, dans cette étude, ont été définies comme des comportements (" manière d'être ou d'agir d'une personne », Centre National de Ressources Textuelles et Lexicales, 2010) que la personne adopte pour parvenir à maintenir un équilibre entre ce qu'elle est et son environnement. Pour les fins de ce projet, nous allons nous concentrer sur les stratégies développées dans le but de gérer la douleur. Celles-ci ont été documentées sur trois plans: 1) l'activité de travail, 2) les requêtes formulées auprès d'autres personnes (autres que travailleurs-euses) pour modifier leur situation de travail et 3) le plan personnel et médical.

La présentation des résultats comporte deux parties principales. La première est consacrée à l'identification et à la description des types de stratégies développées par les travailleuses des usines de transformation du crabe. Cette partie résulte du processus de codage des données recueillies sur le terrain et a permis l'élaboration d'un cadre de référence pour l'étude des stratégies de travailleurs et de travailleuses. Cette partie correspond à l'un des objectifs de cette étude, soit celui d'élaborer et de tester empiriquement un outil pour l'étude des stratégies. La deuxième partie des résultats correspond au second objectif de cette étude, soit celui de décrire la nature et les enjeux des stratégies développées par les travailleuses des usines de transformation du crabe.

\section{1 Élaboration d'un cadre de référence pour l'étude des stratégies}

\subsubsection{Types de stratégies développées : catégories obtenues}

Par l'analyse des données des entretiens et des observations de l'activité de travail pour les saisons de suivi 2005 et 2006, nous avons pu constater que toutes les travailleuses suivies ont développé des stratégies pour gérer leur douleur. Au total, 818 stratégies ont 
été identifiées chez les 16 travailleuses qui ont occupé différents postes. Le nombre de postes occupés est très semblable d'une usine à l'autre, au total 23 à Terre-Neuve avec une moyenne de 2,9 postes (+/- 1,3; max. 5) par travailleuse et 22 sur la Côte-Nord avec 2,8 postes (+/- 1,9; max. 6) par travailleuse. Nous avons pu identifier en moyenne 51,1 stratégies par travailleuse (+/-21,5, min.-max. : 26 à 98).

Ce nombre (818) comprend les stratégies de l'ensemble des travailleuses sur tous les postes occupés. Des stratégies semblables, mais réalisées sur deux postes différents (ou plus) par la même travailleuse, étaient considérées comme des stratégies différentes étant donné que les conditions et les moyens diffèrent d'un poste à l'autre. Il est donc normal que dans nos résultats, une travailleuse ayant occupé plusieurs postes ait développé davantage de stratégies.

Une grande variété de stratégies ressort de l'ensemble des données recueillies. En effet, pour chacune des trois classes de stratégies documentées (activité de travail, requêtes auprès d'autres personnes pour modifier la situation de travail, personnelles et médicales), différents types de stratégies pour gérer la douleur ont été identifiés. Le processus de codage des données a permis de regrouper ces différents types de stratégies sous forme de catégories.

\subsubsection{Cadre de référence pour l'étude des stratégies : Catégories, définitions et exemples de stratégies développées par les travailleuses}

67 Le cadre de référence pour l'étude des stratégies élaboré dans cette recherche est présenté au tableau 1. Les différentes catégories de stratégies obtenues au sein de chaque classe sont présentées, ainsi que des exemples pour chacune des catégories. Ces exemples illustrent la signification et le contenu de la catégorie et mettent ainsi en évidence le caractère empirique de l'outil ancré dans les données recueillies sur le terrain.

Tableau 1. Catégories, définitions et exemples de stratégies développées par les travailleuses pour gérer la douleur

\begin{tabular}{|c|c|}
\hline \multicolumn{2}{|l|}{ Classe : Activité de travail } \\
\hline Définitions & Exemples de stratégies \\
\hline \multicolumn{2}{|l|}{ Catégorie : Opérations } \\
\hline $\begin{array}{l}\text { Façon de s'y prendre pour exécuter chacune des } \\
\text { opérations (physiques et cognitives) de son cycle de } \\
\text { travail. } \\
\text { Englobe tout ce qui a trait aux actions qui concourent à } \\
\text { la réalisation d'une tâche (prescrite et/ou non } \\
\text { prescrite). } \\
\text { Inclut également les opérations que les travailleuses } \\
\text { peuvent ajouter, diminuer en fréquence ou éviter } \\
\text { pendant la réalisation de leur cycle de travail. On peut } \\
\text { aussi parler de réaménagement des opérations, tel que } \\
\text { changer leur ordre d'exécution. }\end{array}$ & $\begin{array}{l}\text { - saisir le crabe près de soi sur la ligne; } \\
\text { - appuyer très légèrement son étampe } \\
\text { sur le tampon d'encre pour ne pas } \\
\text { augmenter ses douleurs aux mains et } \\
\text { bras; } \\
\text { - éviter de peser le crabe en jugeant de } \\
\text { son poids par sa grosseur et la sensation } \\
\text { dans les mains; } \\
\text { - apporter plus d'un panier vide à la fois } \\
\text { à une travailleuse. }\end{array}$ \\
\hline
\end{tabular}


Catégorie : Position du corps et des segments corporels

Façon de positionner son corps et segments corporels par rapport au poste de travail et/ou aux équipements (posture statique ou dynamique).

Ce positionnement peut être par rapport au poste de travail et aux équipements et ne pas être lié à une opération.

Note : cette catégorie pourrait constituer un aspect des modes opératoires (catégorie présentée précédemment), mais la décision a été prise d'en faire une catégorie à part entière pour deux raisons : 1) la posture n'est pas toujours liée à une opération, 2) en raison du nombre élevé de ce type de stratégies élaborées par les travailleuses.

appuyer son corps sur le rebord de la table;

accoter son avant-bras droit sur le rebord du panier;

- utiliser la barre sous la table pour y mettre un pied;

- bouger les jambes et transférer le poids d'une jambe à l'autre.

Catégorie: Outils, équipements, équipements de protection individuel (EPI) et tenue vestimentaire

Regroupe les stratégies mettant en évidence :

1) le choix des outils, des équipements de travail et des équipements de protection individuels (EPI) ;

2) l'utilisation et la non-utilisation des outils, des équipements, ainsi que des EPI ;

3) l'entretien et les ajustements apportés aux outils, aux équipements et aux EPI.

1) Travailler avec la pince à sourcils sans dent plutôt que celle avec dents pour éviter que les morceaux de coquilles restent collés après la pince et ainsi cogner moins souvent et moins fort sur le rebord du bol d'eau (douleur épaules et entre omoplates);

2) Utilisation d'un (ou plusieurs) tapis de caoutchouc pour contrôler les douleurs aux jambes et aux épaules; le port (ou non-port) de gants de coton pour diminuer les douleurs aux mains ;

3) Affiler son couteau pour éviter d'avoir à donner plusieurs coups de couteau sur la pince de crabe pour réussir à la casser.

Catégorie : Aménagement du poste de travail

Actions posées par la personne sur l'emplacement et la disposition des outils et de l'équipement de travail sur et - approcher ses paniers de crabe près autour de son poste de travail et par rapport à la d'elle;

personne (près d'elle, éloigné d'elle, etc.).

Il s'agit d'interventions sur un objet.

- placer son bocal d'eau près du couvercle pour ne pas avoir à s'étirer.

Catégorie : Dimensions temporelles du travail 
Action sur les dimensions temporelles du travail.

Gérer son rythme de travail (plus vite, plus lent).

Profiter d'un arrêt de travail (formel ou informel) ou de production pour pouvoir mieux gérer son travail et son temps.

Agir sur son temps de travail en prenant la décision d'arrêter son travail pour quelques secondes ou minutes (micro-pause et pause) (arrêt informel).

- tenter de commencer sa journée de travail en douceur et augmenter progressivement son rythme de travail pour ainsi «ménager » ses épaules;

- préparer des boîtes à l'avance (le temps que les paniers de crabes défilent dans le bassin de refroidissement) ;

- répartir le nombre de boîtes à faire et à estampiller sur sa journée de travail : si a des douleurs au cours de la journée, arrête un moment de faire des boîtes et ne fait uniquement qu'estampiller les boîtes accumulées.

Catégorie : Interactions entre les travailleuses

\begin{tabular}{|c|c|}
\hline $\begin{array}{l}\text { Action se rapportant aux dimensions collectives de } \\
\text { l'activité : coordination, coaction, coopération et } \\
\text { collaboration, aide et entraide (de la Garza et Weill- } \\
\text { Fassina, } 2000 \text {; Guérin et coll., } 2006 \text {; Caroly, 2010). } \\
\text { Il peut s'agir d'une stratégie qui se met en place } \\
\text { spontanément par une travailleuse envers d'autres } \\
\text { travailleuses ou qui fait suite à une demande explicite } \\
\text { d'une travailleuse envers une autre travailleuse. }\end{array}$ & $\begin{array}{l}\text { - partager les crabes avec sa voisine; } \\
\text { - demander à sa collègue de l'aider à } \\
\text { soulever un panier de crabe rempli; } \\
\text { - tenir les rabats de la boîte pendant } \\
\text { qu'une autre travailleuse dépose le bloc } \\
\text { de capelans dans la boîte; } \\
\text { - décider d'emballer une autre grosseur } \\
\text { de crabes pour alléger la charge de } \\
\text { travail d'une collègue; } \\
\text { - demander à la travailleuse du poste en } \\
\text { amont de ne pas mettre le plastique sous } \\
\text { le panier. }\end{array}$ \\
\hline
\end{tabular}

Catégorie : Rotation

Action d'organiser une rotation entre des postes de travail selon un ordre cyclique et un rythme pré-établi (Vézina, 2003).

- la rotation au département de la chair entre cinq postes de travail ;

Les travailleuses décident, entre elles, de faire de la rotation, car cela les aident à gérer leur douleur.

- la rotation à l'emballage et au poste de faire des boites entre deux travailleuses.

Catégorie : Relocalisation

Action de changer de poste (dans le même département ou un autre département).

Ce changement peut être temporaire ou permanent et a été effectué par la travailleuse elle-même.

- travailleuse travaille au poste de la scie pour un moment (deux heures), car ses douleurs aux épaules étaient trop importantes pour poursuivre à l'empaquetage ;

- travailleuse qui décide de changer de place sur la ligne en raison $d u$ froid (courant d'air venant du congélateur) qui augmentait ses douleurs.

Catégorie : Apprentissages 


\begin{tabular}{|c|c|}
\hline $\begin{array}{l}\text { "S'instruire ", découvrir de nouvelles façons de faire } \\
\text { pour mieux gérer ses douleurs. } \\
\text { Concerne le partage de trucs par des échanges entre } \\
\text { collègues de travail ou en entendant la conversation } \\
\text { d'autres travailleuses sur leurs douleurs et leurs façons } \\
\text { de les gérer. }\end{array}$ & $\begin{array}{l}\text { - au poste de mirer la chair, en discutant } \\
\text { avec une collègue de travail, une } \\
\text { travailleuse ayant mal au dos apprend } \\
\text { qu'elle peut procéder autrement et place } \\
\text { son bol à tel endroit par rapport à elle } \\
\text { pour diminuer ses douleurs à l'épaule. }\end{array}$ \\
\hline \multicolumn{2}{|l|}{ Catégorie : Contrôle de soi } \\
\hline $\begin{array}{l}\text { Actions par lesquelles les travailleuses parviennent à } \\
\text { faire abstraction de leur douleur afin de réussir à la } \\
\text { gérer. } \\
\text { Comprend les stratégies qui se traduisent dans l'activité } \\
\text { par une forme d'autorégulation de soi afin de détourner } \\
\text { son attention de la douleur (Durand et coll., 2008). }\end{array}$ & $\begin{array}{l}\text { - parler de ses douleurs avec une collègue } \\
\text { de travail; } \\
\text { - parler en travaillant dans le but de se } \\
\text { changer les idées et de ne pas penser à } \\
\text { ses douleurs; } \\
\text { - se donner des défis ou s'organiser des } \\
\text { "jeux» en travaillant pour réussir à } \\
\text { oublier la douleur. }\end{array}$ \\
\hline \multicolumn{2}{|c|}{$\begin{array}{l}\text { Classe : Requêtes auprès d'autres personnes afin qu'il y ait modification de leur situation de } \\
\text { travail }\end{array}$} \\
\hline Définitions & Exemples de stratégies \\
\hline \multicolumn{2}{|l|}{ Catégorie : Demandes aux superviseurs } \\
\hline $\begin{array}{l}\text { Démarche entreprise par une travailleuse envers un/une } \\
\text { superviseur(e) ou contremaître afin d'apporter une } \\
\text { amélioration à sa situation de travail. }\end{array}$ & $\begin{array}{l}\text { - demander à un superviseur pour } \\
\text { changer de poste; } \\
\text { - demander au superviseur d'aviser le } \\
\text { travailleur du quart de travail précédent } \\
\text { au même poste afin que les piles de } \\
\text { paniers de crabe ne soient pas trop } \\
\text { hautes; } \\
\text { - aviser le superviseur de ne plus vouloir } \\
\text { retourner travailler à tel poste. }\end{array}$ \\
\hline \multicolumn{2}{|l|}{ Catégorie : Demandes aux ressources humaines ou à l'entreprise } \\
\hline $\begin{array}{l}\text { Démarche entreprise par une travailleuse envers une } \\
\text { personne responsable des ressources humaines ou qui } \\
\text { fait partie de la direction de l'entreprise. }\end{array}$ & $\begin{array}{l}\text { - aviser la personne responsable des } \\
\text { ressources humaines qu'un poste était } \\
\text { trop exigeant pour une femme, car le } \\
\text { travailleur supposé faire telle partie du } \\
\text { travail ne la faisait pas et elle devait la } \\
\text { faire elle-même; } \\
\text { - soumettre sa candidature pour un } \\
\text { autre poste en raison des douleurs } \\
\text { ressenties au poste occupé. }\end{array}$ \\
\hline & \\
\hline
\end{tabular}




\begin{tabular}{|c|c|}
\hline $\begin{array}{l}\text { Démarches ou sollicitations entreprises par une } \\
\text { travailleuse envers les personnes préposées à l'entretien } \\
\text { et à la réparation de l'équipement et du matériel de } \\
\text { travail. }\end{array}$ & $\begin{array}{l}\text { - discuter avec la maintenance de la } \\
\text { possibilité de modifier la hauteur du } \\
\text { convoyeur, car trop élevée et cause des } \\
\text { douleurs aux épaules; } \\
\text { - informer la maintenance que les } \\
\text { rouleaux sur lesquels la travailleuse } \\
\text { pousse les boites ne roulent pas bien et } \\
\text { cette situation contraint à pousser plus } \\
\text { fort, ce qui augmente les douleurs aux } \\
\text { épaules. }\end{array}$ \\
\hline \multicolumn{2}{|l|}{ Classe : Personnelle et médicale } \\
\hline Définitions & Exemples de stratégies \\
\hline \multicolumn{2}{|l|}{ Catégorie : Médication } \\
\hline Prise d'un médicament prescrit ou non prescrit. & $\begin{array}{l}\text { - prise d'anti-inflammatoires ayant été } \\
\text { prescrits par un médecin au début de la } \\
\text { saison; } \\
\text { - prise d'une substance analgésique } \\
\text { (Tylenol, etc.). }\end{array}$ \\
\hline \multicolumn{2}{|l|}{ Catégorie : Traitements } \\
\hline $\begin{array}{l}\text { Consultation et/ou administration d'un soin de santé } \\
\text { par un professionnel de la santé. }\end{array}$ & $\begin{array}{l}\text { - consulter son médecin avant le début } \\
\text { de la saison; } \\
\text { - séance de massage à la fin de la saison } \\
\text { de travail. }\end{array}$ \\
\hline \multicolumn{2}{|l|}{ Catégorie : Organisation à la maison } \\
\hline $\begin{array}{l}\text { Organisation des tâches domestiques et de la vie } \\
\text { familiale. }\end{array}$ & $\begin{array}{l}\text { - conjoint aide aux tâches ménagères; } \\
\text { - conjoint s'occupe d'aller chercher les } \\
\text { enfants à la garderie; } \\
\text { - ne pas faire de ménage durant la } \\
\text { saison; } \\
\text { - préparer des repas à l'avance ou } \\
\text { acheter des repas déjà préparés. }\end{array}$ \\
\hline & \\
\hline
\end{tabular}


Concerne les loisirs, l'activité physique, les méthodes de relaxation ou les soins personnels réalisés dans un but de diminuer la douleur.
- aller prendre une marche après la journée de travail ;

- se coucher sur un coussin chauffant (coussin hauteur bas $\mathrm{du}$ dos) pour dormir :

- prendre un bain chaud en arrivant de travailler;

- frotter ses épaules avec de la crème ;

- s'asseoir et relaxer.

Catégorie : Gestion du temps qui lui « appartient » pendant le temps de travail et en dehors du travail

Action de gérer le temps disponible pendant le travail et en dehors du travail afin de contrôler la douleur.

Met en relation la dimension temporelle du travail et l'aspect personnel.

D'une part, ces stratégies sont élaborées lors d'un arrêt de travail (formel ou informel). D'autre part, c'est au cours de cet arrêt de travail que la travailleuse en profite pour s'occuper d'elle et de ses douleurs. Les deux aspects (personnel et dimension temporelle) sont présents au sein de la stratégie pour classer celle-ci sous cette catégorie.

Note: Contrairement à la catégorie «dimensions temporelles du travail» où la travailleuse agit pour pouvoir mieux gérer son travail et son temps (ex.: préparer des boîtes à l'avance), les stratégies de la catégorie «utilisation du temps qui lui appartient pendant le travail et en dehors du travail » consistent à utiliser son temps à des fins personnelles (s'étirer, se masser, etc.) pour gérer la douleur.

- prendre la décision d'arrêter son travail quelques secondes ou minutes ou profiter d'un arrêt de production (pas de crabes sur le convoyeur) pour s'étirer, se frotter, s'asseoir, etc.

- profiter de son heure de dîner pour aller manger chez sa mère, car cela lui permet de vraiment relaxer et relâcher ses épaules;

- prendre une pause toilette pour s'asseoir et relaxer ses épaules;

- profiter d'une journée sans production pour relaxer et ainsi ne pas augmenter ses douleurs.

Catégorie : Attitude face à la douleur

Disposition d'esprit, façon de voir ou représentation de la douleur qui amène la travailleuse à orienter son comportement en fonction de cette représentation.

- travailleuse souligne " tenter d'ignorer

Pour les fins de cette recherche, le terme « attitude » est interprété comme une « disposition d'esprit, déterminée par l'expérience à l'égard d'une personne, d'un groupe social ou d'une chose abstraite (problème, idée, «s'habituer à sa douleur et vivre avec doctrine, etc.) et qui porte à agir de telle ou telle celle-ci». manière » (Centre National de Ressources Textuelles et Lexicales, 2010).

sa douleur et de fonctionner à travers celle-ci»; une autre travailleuse mentionne 
La catégorisation obtenue permet de constater l'étendue et la variété des types de stratégies développées par les travailleuses pour gérer leur douleur et parvenir à demeurer au travail. Cette grande variété s'étend des dimensions qui relèvent uniquement de la travailleuse à des dimensions faisant ressortir des interactions avec différents acteurs du milieu de travail, jusqu'à des dimensions qui débordent du cadre du travail et qui interpellent l'entourage proche de la travailleuse.

\subsection{Nature et enjeux des stratégies développées par les travailleuses}

Dans cette deuxième partie des résultats, il sera question de la nature et des enjeux des stratégies développées par les travailleuses suivies. Les verbatim des travailleuses expliquant leurs stratégies seront utilisés pour illustrer différentes thématiques.

\subsubsection{Des stratégies pour gérer la douleur afin de se maintenir au travail}

70 Les résultats de cette étude permettent de constater que toutes les travailleuses suivies ont effectivement recours à l'élaboration de stratégies pour gérer leur douleur. Pour ces travailleuses, il s'agit d'endurer, de « se pousser pour passer au travers ». Les travailleuses gèrent et endurent leur douleur en se disant que ce n'est que temporaire, le temps de la saison, le temps d'accumuler les heures pour être éligibles à l'assurance-emploi. Comme l'illustrent les propos d'une des travailleuses de l'usine de la Côte-Nord (travailleuse 14), on endure, car on sait que c'est une « claque à donner » :

Travailleuse : «Ah! mon médecin m’a appelée en fin de semaine elle était enragée ! Elle trouve que ça n'a pas d'allure. Bien j'ai dit : « Regarde je me sens bien. »

Chercheuse : "Puis qu'est-ce qu'elle t'a conseillé ? »

Travailleuse : « 40 heures et moins. »

Chercheuse : «Ok, puis toi tu as fait 85 heures la semaine dernière ? »

Travailleuse : « Regarde c'est une claque à donner, je le sais... »

\subsubsection{Des enjeux pour la santé}

71 La nature de certaines des stratégies développées soulève quelques interrogations quant aux impacts et aux coûts qu'elles représentent pour la santé. D'un côté, certaines stratégies semblent bénéfiques à la fois pour gérer la douleur et sur le plan de la santé du moins à court et moyen terme. À titre d'exemple, la stratégie développée par l'une des travailleuses (travailleuse 11), qui consiste à échanger de poste avec une travailleuse à toutes les deux heures, met en évidence les bienfaits que celle-ci apporte.

«C'est moins répétitif, c'est moins... Même si ça a l'air de rien, juste de changer de côté comme ça à toutes les deux heures avec Julie [nom fictif d'une travailleuse] là, dans le fond le mouvement n'est pas beaucoup différent, mais il est vraiment différent. [...] Quand j'ai eu fait plusieurs journées là sans changer, je me suis aperçue que j'avais les mêmes douleurs [que sa collègue] qui se développaient. Fait que j'ai dit: "Cette année on ne va pas attendre d'avoir mal, on va changer tout de suite aux deux heures. » Parce que l'année passée on retardait tout le temps, on changeait quand on avait mal. Mais quand la douleur est installée là, hein ! [...]» 
72 C'est dans ce même ordre d'idées que des travailleuses insistent sur l'importance d'alterner de bras quand elles travaillent. Notamment, l'une des travailleuses de l'usine de Terre-Neuve (travailleuse 1) mentionne :

«Tu ne fais pas les mêmes mouvements répétitifs tout le temps, tu changes la façon dont tu travailles. Tu n'utilises pas toujours les mêmes muscles et... Parce que de différents côtés ça aide aussi. Habituellement, la plupart du temps, je les prends... avec ma main gauche. Quand je travaille sur les 10 plus [grosseur de crabe] et à la scie, la majorité du temps, je les saisis avec ma main droite, donc tu donnes une pause à ton autre épaule. De plus, tu changes de main, donc tu ne saisiras pas toujours avec la même main. J'essaie d'alterner de main, car ce qui m'est arrivé quand je me suis blessée à l'épaule était de toujours atteindre avec ma main droite. » (notre traduction)

73 On peut se questionner sur ce type de stratégies lorsque utilisé à plus long terme. L'alternance des bras peut en effet permettre un repos régulier de certaines structures musculo-squelettiques et d'éviter l'avenue de la douleur. Cependant, il est important de considérer, d'une part, que dans des conditions extrêmes cette stratégie apporte de toute façon une usure du côté gauche et du côté droit et, d'autre part, que même si certaines structures alternent, d'autres peuvent être toujours sollicitées, les muscles proximaux (cou, haut du dos), par exemple, versus les muscles distaux (mains, avant-bras), dépendant des tâches à réaliser.

74 Par ailleurs, d'autres travailleuses qui ont développé de la douleur d'un côté du corps, le poignet droit, par exemple, développent la stratégie d'utiliser seulement le côté le moins douloureux. Saisir le crabe avec le bras le moins douloureux sans alternance des bras peut être une stratégie efficace pour gérer la douleur dans l'immédiat, mais ne plus être d'aucune utilité pour diminuer la douleur après un certain temps. Et même parfois devenir néfaste ou à risque pour la travailleuse. Après une certaine période de temps, il est possible que cette stratégie provoque, au rebond, une apparition ou une augmentation des douleurs au bras a priori le moins affecté.

\subsubsection{Des compromis nettement coûteux pour la santé}

Bien que ces stratégies soient élaborées pour gérer la douleur, certaines d'entre elles peuvent effectivement représenter des compromis coûteux pour la santé. Nul doute qu'une stratégie telle qu'ignorer sa douleur peut entraîner des répercussions non négligeables sur la santé :

«Quelques fois tu ignores que ça fait mal, tu essaies juste de l'ignorer. Tu ignores! Et quelques fois, je dois tirer fort sur le plastique et cela fait un peu plus mal, tu sais comme un rapide... mais après disons que tu n'es plus capable d'endurer, tu dis : « ouch! ». Mais tu fais juste l'ignorer et tu continues parce que tu sais que tu dois le faire! Tu n'as pas d'autre choix ! (notre traduction, verbatim TN3)

Dans le même sens, prendre des anti-inflammatoires permet de diminuer la douleur pour une certaine durée, mais n'est pas sans conséquence après une consommation prolongée. Plusieurs travailleuses suivies ont mentionné ne plus recourir à cette stratégie, ou du moins tenter de limiter leur consommation en raison des conséquences engendrées par une surconsommation dans le passé. 


\subsubsection{Une prise de conscience} par les travailleuses mettent en évidence leur habileté, leur imagination et leur savoirfaire. À titre d'exemple, la stratégie développée par l'une des travailleuses (travailleuse 7), qui consiste à laisser tremper les pinces de crabe quelques minutes dans l'eau tiède, lui permet de réussir à casser la pince en donnant moins de coups de couteau et en cognant moins fort. Cette travailleuse rapportait qu'affiler son couteau l'aidait également, mais que sans le trempage des pinces, son épaule était beaucoup plus douloureuse à la fin de sa journée de travail. d'estampiller les cartons. Celle-ci rapporte que dès qu'elle sent devoir exercer une pression plus forte avec son étampe sur le pad d'encre, elle ajoute de l'encre dans celui-ci. Ainsi, elle dit s'éviter de devoir appuyer fort avec sa main, ce qui lui cause des douleurs à la main et au coude.

83 Un autre exemple est celui de la travailleuse de l'usine de la Côte-Nord qui recueille les paniers de crabe des empaqueteuses pour les transporter avec un chariot au poste de pesage. Cette travailleuse souligne déposer sur son chariot deux paniers de crabes des empaqueteuses de la table basse en premier et ensuite, déposer sur ces paniers deux 
paniers des empaqueteuses de la table haute pour être en mesure de pouvoir glisser les paniers sur le chariot et ne pas les soulever.

C'est dans ce même ordre d'idées que des travailleuses au poste d'empaquetage rapportent saisir la section de crabe la plus proche d'elles ou encore approcher leurs paniers près d'elles pour ne pas avoir à s'étirer. À cet égard, l'une des travailleuses de l'usine de la Côte-Nord (travailleuse 9) mentionne :

«Prendre ce qui passe en avant de toi le plus possible pour ne pas étirer tes bras trop loin... pourquoi aller les chercher plus loin?»

Selon cette travailleuse, cette façon de travailler, c'est-à-dire ne pas s'étirer ou en d'autres termes, faire des mouvements dans sa zone d'atteinte, représente une façon de travailler avantageuse, mais la stratégie consiste aussi à se regarder travailler, à prendre conscience de sa posture :

«Puis surtout là le truc le mieux c'est de travailler proche de ton corps, ça là je te le jure, c'est vraiment un truc là je me dis, ça commence, puis travaille proche là, puis ça roule si vite de toute manière, parce que vu qu'il y en a toujours qui passe je n'ai pas à aller loin. Souvent

«Oups! Arrête d'aller loin! Il y en a proche, pourquoi tu vas loin?»

C'est parce que des fois ton regard va plus loin. Des fois je n'y pense pas, mais quand j'ai du mal j'y pense parce que je me dis :

«Approche ton panier parce que là ça revient, là Andrée [nom fictif de la travailleuse], tu commences à avoir mal à l'épaule, approche tes paniers puis là fais-le... » ».

86 Ces quelques exemples font ressortir non seulement la grande connaissance et les savoirfaire qu'ont développés les travailleuses par rapport à leur travail, mais également leur ingéniosité d'esprit pour parvenir à élaborer des façons de faire avec les moyens dont elles disposent afin de gérer leur douleur.

\section{Discussion}

87 Cette discussion comportera quatre parties principales. Premièrement, des enjeux de santé autour du développement des stratégies chez le groupe de travailleuses étudié seront soulevés. Nous discuterons ensuite de l'apport du cadre de référence élaboré au cours de ce projet en ergonomie pour l'étude des stratégies, pour en troisième lieu, discuter de ses assises dans l'approche développée par Yin sur les études de cas. Les limites de cette étude termineront cette discussion.

\subsection{Gérer la douleur pour se maintenir au travail}

Les résultats permettent de constater que les travailleuses de l'industrie de la transformation du crabe développent effectivement des stratégies pour gérer leur douleur. La mise à plat des stratégies fait ressortir les bienfaits que semblent apporter certaines d'entre elles aux travailleuses. Des stratégies telles que « approcher les paniers de crabe près de soi », « affiler son couteau pour éviter d'avoir à donner plusieurs coups » ou encore « utiliser la barre sous la table pour y mettre un pied et transférer le poids du corps d'une jambe à l'autre » constituent quelques exemples de stratégies élaborées par les travailleuses pour réguler leur travail tout en se préservant, ou du moins en 
diminuant les risques de TMS. L'élaboration de stratégies de préservation de la santé a également été abordée dans de nombreux travaux (Caroly, 2010 ; Ouellet et Vézina, 2008 ; Premji et coll., 2008 ; Chassaing, 2006 ; Toupin, 2005 ; Pueyo et Gaudart, 2000 ; Cloutier et coll., 2005 ; 1998 ; Gaudart, 1996 ; Cloutier, 1994). Ces travaux font ressortir les capacités des travailleurs à se construire des marges de manœuvre dans le but de préserver leur santé tout en répondant aux exigences de production.

\subsubsection{Quand les moyens pour gérer sa douleur deviennent des compromis coûteux pour sa santé : les limites des stratégies}

Bien que les résultats permettent de constater les bénéfices associés à plusieurs stratégies à la fois pour gérer la douleur et sur le plan de la santé, d'autres résultats laissent transparaître que les stratégies élaborées par les travailleuses peuvent également présenter d'importantes limites et même exposer les travailleuses à des risques pour leur santé. En effet, rappelons que l'objectif des stratégies développées par les travailleuses dans ce projet est de gérer la douleur pour être en mesure de se maintenir au travail pendant la saison du crabe. Ceci ne correspond pas toujours à la recherche d'un équilibre entre leur santé et les exigences du travail.

Par exemple, les stratégies peuvent présenter des limites dans le temps. Prendre des antiinflammatoires permet de diminuer la douleur pour une certaine période, mais n'est pas sans conséquence après une consommation prolongée. Les propos des travailleuses font d'ailleurs ressortir les conséquences dans le temps et ne peuvent être la solution définitive au problème. Les stratégies peuvent aussi déplacer le problème d'une région du corps à l'autre. Saisir le crabe sur le convoyeur avec le bras le moins douloureux permet effectivement de gérer la douleur, mais après une certaine période de temps, peut s'avérer être à risque de développement de TMS dans une autre région.

Outre ces dernières limites, les stratégies peuvent également présenter des limites selon les individus. Une travailleuse ayant des douleurs à l'épaule n'adoptera pas les mêmes stratégies qu'une travailleuse éprouvant des douleurs au poignet. Dans le même sens qu'une petite travailleuse ayant développé comme stratégie d'utiliser quatre tapis un pardessus l'autre au sol afin de ne pas avoir à trop étirer ses bras lorsque vient le temps de saisir un panier pourrait constituer une stratégie à risque pour sa collègue de travail de grande taille. Ainsi, une stratégie peut être bénéfique pour une travailleuse, mais à risque pour une autre.

De plus, il n'y a pas de doute que trouver des stratégies pour oublier qu'on a mal comporte des risques. Comme nous l'avons souligné, les stratégies sont développées par les travailleuses pour se maintenir au travail et n'ont pas toujours le potentiel de prévenir ou du moins de ne pas aggraver les TMS. En ce sens, il est risqué, dans un objectif de prévention, de se reposer uniquement sur les capacités des travailleuses à se construire des marges de manœuvre. L'identification de déterminants à l'origine des difficultés rencontrées par les travailleuses a d'ailleurs permis d'améliorer certaines situations de travail au cours de l'intervention menée en parallèle à cette recherche.

Conditionnées à la fois par les objectifs de production et de l'organisation du travail, ainsi que par les caractéristiques personnelles des travailleurs, les stratégies constituent des compromis qu'endossent les travailleurs pour parvenir à leurs fins (Bourgeois et coll., 2006 ; 2000). Comme le soutiennent ces auteurs, la connaissance du contexte de la situation est essentielle à la compréhension des stratégies. 


\subsubsection{Travail saisonnier et gestion des douleurs}

usines de crabe de ce projet est l'élaboration d'une catégorisation des stratégies. Cette catégorisation se situe dans le prolongement des travaux de Guérin et coll. (2006) en proposant un cadre de référence facilitant la mise en évidence des stratégies et la compréhension du travail.

97 En adoptant une approche d'immersion de la chercheuse dans le milieu de travail pendant plusieurs semaines dans deux milieux de travail et sur deux saisons, en multipliant les modes de recueil de données pour mettre en évidence les stratégies (observation de l'activité de travail et verbalisations au poste de travail, entretiens, retour sur les schémas corporels, rencontres d'autoconfrontation), nous avons pu découvrir un nombre impressionnant de stratégies malgré le fait que les travailleuses occupent des postes de travail offrant peu de marge de manœuvre.

En particulier par le fait de couvrir deux saisons au cours desquelles la chercheuse s'est intéressée à l'expression des travailleuses sur leurs stratégies pour gérer la douleur, ces travailleuses ont acquis une plus grande conscience de leur mode opératoire et ont développé leur capacité à rendre compte de leur activité de travail et des raisons qui sous-tendent leur façon de faire. En fait, dès la première saison, les travailleuses avaient 
déjà rempli leurs schémas corporels quotidiens en réfléchissant si elles avaient développé des stratégies pour gérer leur douleur. Durant la période entre les deux saisons, la chercheuse avait donc déjà une importante banque de stratégies qui lui a fourni l'opportunité de développer un premier système de classement (grille, point $\mathrm{C}$, figure 1). Ceci a donc permis à la chercheuse elle-même d'aller plus loin dans son questionnement lors de la deuxième saison avec les 16 travailleuses et de parvenir à la proposition de ce cadre de référence pour le recueil et l'analyse des stratégies.

La catégorisation résultant de cette étude constitue un cadre méthodologique spécifique à la documentation des stratégies. Ceci représente un apport pour l'étude des TMS en constituant une grille pour l'étude des stratégies développées par les travailleurs et les travailleuses. Très peu d'outils de ce type semblent être à la disposition des chercheurs et des ergonomes.

\subsubsection{Un cadre de référence pour faciliter les verbalisations}

100 Comme l'a proposé Vermersch (1991), il y a intérêt à développer des moyens pour faciliter l'expression des personnes sur leur activité de travail. L'une des conditions qu'il propose pour favoriser la description d'activités réelles et pour faire émerger les compétences et les connaissances implicites mises en œuvre dans des situations concrètes est de distinguer des catégories. Ces catégories constituent une des conditions nécessaires pour que l'interviewé puisse rendre compte le plus finement possible de ce qu'il a fait réellement et de comment il s'y est pris pour faire ce qu'il avait à faire. Parvenir à expliciter ses méthodes ou encore la « mise en mot » constitue, d'après Vermersch (1991), l'un des outils les plus puissants à la prise de conscience du sujet. Ce dernier devient progressivement en mesure de prendre conscience de son activité et des fondements personnels de cette manière d'agir en situation, pour mieux le vivre ou le modifier.

101 En ce sens, le cadre de référence proposé pour l'étude des stratégies se veut un guide pour le chercheur et l'ergonome: d'une part, pour établir des dimensions précises à investiguer lors des observations; d'autre part, pour raffiner le questionnement des travailleurs en vue d'accéder à des dimensions du vécu qui ne sont pas immédiatement présentes à la conscience de la personne et ainsi obtenir des données d'une plus grande richesse et profondeur.

\subsubsection{Débat sur le potentiel des stratégies à préserver la santé et partage des savoirs}

102 En mettant à jour l'éventail des stratégies développées par les travailleuses, cette catégorisation offre la possibilité d'une mise en débat sur le fait que les contraintes liées au travail peuvent entraîner le développement de stratégies permettant de gérer la douleur, mais pas nécessairement de se maintenir en santé. De plus, nombre de stratégies peuvent servir de base à un partage des savoirs pouvant être intégrés à une formation. En ce sens, cette étude poursuit la lignée des travaux réalisés par Vézina et coll. (1999), Chatigny et coll. (2003) et Ouellet (2009) sur le plan de la prévention dans les milieux de travail. La mise à plat des stratégies combinée à leur classement permet une conscientisation des travailleurs-euses à leurs façons de faire et facilite d'autant plus la transmission des savoirs d'une travailleuse à l'autre et le développement des savoir-faire. 


\subsection{Un outil méthodologique pour l'étude des stratégies dont les assises reposent sur le respect des critères de scientificité} mesures opérationnelles adéquates aux concepts étudiés»(Yin, 2003, p. 34) a été respecté, entre autres, par le biais du recours à des sources d'évidences multiples (observations de l'activité et de l'organisation du travail, divers types d'entretiens). La convergence des méthodes contribue à consolider la validité du processus (Yin, 2003) permettant une démonstration plus précise, plus convaincante et, par conséquent, d'une plus grande qualité scientifique. De plus, la triangulation des modes de collecte de données a permis non seulement de mettre en comparaison mais également en complémentarité les données obtenues au moyen de chacun de ces modes de collecte. De leur côté, les validations entre chercheurs ont permis de s'assurer de la justesse de chacune des catégories, ainsi que de leur définition en apportant un regard extérieur sur ce processus. Comme le souligne Savoie-Zajc (2003), l'objectivité recherchée à travers ce processus est assurée par la triangulation de la subjectivité des chercheurs. De plus, tel que recommandé par Yin (2003, p. 34), une "chaîne de l'évidence » a été établie par la rédaction d'un protocole de recherche assurant la cohérence entre la question de recherche, la recension des écrits, la collecte des données, leur analyse et les résultats produits. Ce protocole avait été approuvé préalablement par un jury de thèse (proposition de recherche : Major, 2005). 


\subsubsection{Fiabilité}

107 Pour s'assurer de la fiabilité des résultats, c'est-à-dire «la démonstration que les différentes phases du processus de recherche, notamment la collecte des données, peuvent être répétées et conduire aux mêmes résultats " (Yin, 2003, notre traduction, p. 34), trois principales procédures ont été suivies. Yin (2003, notre traduction, p. 37) souligne que l'accent est mis sur le même cas et non sur la "réplication » des résultats d'un cas en faisant une autre étude de cas. L'une de ces procédures consistait en l'élaboration et le suivi du protocole de recherche qui documentait toutes les étapes, assurant ainsi la traçabilité du processus (Yin, 2003). Une seconde procédure fut l'élaboration d'une banque de données regroupant l'ensemble des stratégies identifiées sur le terrain au cours des deux années de suivi pour l'ensemble des travailleuses donnant ainsi accès à une éventuelle investigation du processus de recherche. La mise en contexte des résultats obtenus a été assurée par la tenue d'un journal de bord par la chercheuse tout au long de l'étude.

\subsubsection{Validité externe}

108 généralisables » (validité externe) (Yin, 2003, p. 34) repose en grande partie sur le choix de recourir à un devis d'étude de cas multiples plutôt que simple et sur la logique de réplication qui sous-tend cette étude de cas multiples. Le choix d'avoir sélectionné des sujets provenant de deux usines distinctes et occupant différents postes offre une validité externe aux résultats beaucoup plus importante que si un seul cas avait été étudié et si l'ensemble des cas provenaient de la même usine et tous du même poste. Cette logique de réplication théorique où des cas globalement semblables (vivant tous des épisodes de douleur), mais contrastant sur des dimensions prédéterminées (usines, postes), contribue à la robustesse des résultats obtenus et permet de décrire adéquatement et de façon davantage exhaustive le phénomène observé (Eisenhardt, 1989 ; Yin, 2003).

La validité externe du cadre de référence obtenu pour l'étude des stratégies se distingue sur deux dimensions : 1) la dimension selon laquelle l'outil élaboré peut être utilisé pour étudier les stratégies développées par les travailleurs-euses dans différents milieux de travail; 2) la dimension selon laquelle cette catégorisation peut servir d'outil méthodologique sur lequel les recherches futures pourront prendre appui pour l'étude de l'activité de travail et la genèse des TMS. Ce type de généralisation dont il est question dans les études de cas correspond à la généralisation analytique (Yin, 2003). Cette généralisation repose sur le principe que l'ensemble particulier des résultats produits par l'étude de cas peut être généralisé à une théorie plus large qui décrit adéquatement le phénomène observé (Eisenhardt, 1989 ; Yin, 2003). Dans cette étude, chaque cas a permis de fortifier, d'enrichir et d'affiner cette catégorisation. Les catégories et les définitions obtenues sont ancrées empiriquement dans des données terrain, recueillies sur une période de deux années, dans deux usines distinctes et provenant de 16 cas occupant une variété de postes de travail. À cet égard, la catégorisation obtenue laisse présager d'une validité externe suffisante pour servir de point de départ à l'étude des stratégies mises en œuvre par les travailleurs-euses dans d'autres milieux de travail, et ce, à titre d'outil méthodologique pour des projets d'intervention et de recherche. 


\subsection{Limites de l'étude}

110 Bien que cet outil ait été élaboré et testé empiriquement auprès de 16 travailleuses occupant différents postes et au sein de deux usines situées dans deux provinces différentes, il n'en demeure pas moins que les catégories émergentes des classes « activité de travail » et « requêtes auprès d'autres personnes » découlent de la nature du travail réalisé dans ces usines, soit un milieu où le travail manuel et répétitif se fait à la châne. Toute nouvelle étude permettra sûrement de raffiner les catégories de stratégies en particulier dans les milieux où le travail n'est pas manuel. Il est probable qu'une analyse des stratégies développées par des travailleurs et des travailleuses dont le travail relève, par exemple, d'une relation de service pourrait entraîner l'émergence de catégories communes à celles obtenues, mais également de catégories spécifiques à l'aspect du travail relationnel et affectif caractérisant l'activité de travail en relation de service (Cloutier et coll., 2005; Teiger et coll., 2005; Caroly, 2010). En ce sens, ce cadre de référence peut constituer un point de départ auquel de nouvelles catégories peuvent être ajoutées ou encore des catégories pourraient être divisées en sous-catégories afin de correspondre plus finement à un secteur en particulier.

111 Le cadre de référence élaboré dans cette étude prétend articuler l'ensemble du contenu des données obtenues et être ancré dans les données recueillies sur le terrain. Il se veut également un modèle pour d'autres recherches qui pourront utiliser, dans une démarche déductive, les catégories obtenues et ajouter à cette liste, dans une démarche inductive, les particularités relevant de leur milieu et de leur contexte (syndiqué ou non, petite vs grosse entreprise, etc.).

\section{Conclusion}

112 En conclusion, les résultats de cette étude permettent de constater que les travailleuses suivies, qui proviennent de deux usines de transformation du crabe dont l'une est située au Québec et l'autre à Terre-Neuve, élaborent une grande variété de stratégies pour gérer leur douleur. L'immersion de la chercheuse dans ces milieux sur une période relativement longue, soit un total de 20 semaines sur trois ans (incluant l'étude pilote), a rendu possible l'obtention d'une compréhension détaillée de la situation à l'étude et d'un corpus inédit de données. La démarche itérative de la collecte et de l'analyse des données a mené à l'élaboration d'un cadre de référence pour l'étude des stratégies des travailleurs et des travailleuses. Ce cadre de référence élaboré de manière inductive est ancré empiriquement dans les données recueillies sur le terrain et possède un caractère intégrateur en ce sens qu'il prend en compte l'ensemble des données obtenues. La logique de réplication théorique qui sous-tend cette étude contribue également à la force de ce cadre de référence. Selon nous, l'élaboration d'un tel cadre de référence pour l'étude des stratégies constitue une avancée pour les connaissances en ergonomie. Les chercheurs et les ergonomes disposeront d'un outil pour l'identification et le classement des stratégies et pourront y ajouter d'autres catégories selon les caractéristiques des milieux étudiés.

Ce cadre de référence rend compte des différents types de stratégies élaborées par les travailleuses sur le plan de l'activité de travail, des requêtes auprès d'autres personnes pour modifier leur situation, ainsi que sur le plan personnel et médical. Les travailleuses suivies ont recours à une telle gestion de la douleur pour parvenir à se maintenir au 
travail afin d'accumuler le nombre d'heures requis par le système d'assurance-emploi. Les résultats obtenus permettent de constater que certaines des stratégies présentent toutefois d'importantes limites et semblent représenter des compromis coûteux pour leur santé. Ces résultats mettent en évidence les capacités dont disposent les travailleuses, mais laissent également présager de la présence d'importantes contraintes qui pourraient influencer le type de stratégies auxquelles les travailleuses ont recours, ainsi que l'impact de ces stratégies sur la santé des travailleuses. En ce sens, il serait primordial de documenter les facilitateurs ainsi que les barrières à l'élaboration de stratégies par les travailleuses et d'investiguer les indicateurs permettant de juger jusqu'à quel point une stratégie est saine pour la santé ou, au contraire, expose la travailleuse à des risques dont ceux de TMS.

Les auteures tiennent à exprimer leurs plus sincères remerciements aux travailleuses ainsi qu'aux entreprises de Terre-Neuve et du Québec ayant participé à cette étude. Les auteures remercient également SafetyNet, le CINBIOSE, ainsi que Pierre Chrétien et Claire MacGillivray du réseau de la santé publique de la Côte-Nord pour leur soutien. Cette étude a été rendue possible grâce au soutien financier de l'Institut de recherche Robert-Sauvé en santé et en sécurité du travail (IRSST), de l'Institut Santé et société (ISS, bourse AON), du Centre de recherche interdisciplinaire sur la biologie, la santé et l'environnement (CINBIOSE) et du Réseau de recherche en santé et en sécurité du travail du Québec - axe TMS (RRSSTQ-axe TMS). Les auteures demeurent seules responsables du contenu de cet article.

\section{BIBLIOGRAPHIE}

Armstrong T.J., Foulke J.A., Joseph B.S., Goldstein S.A. (1982). Investigation of cumulative trauma disorders in a poultry processing plant. American Industrial Hygiene Association Journal, vol. 43, $\mathrm{n}^{\circ} 2$, p. 103-116.

Authier, M. (1996). Analyse ergonomique des stratégies de manutentionnaires experts et novices. Thèse de doctorat. Université de Montréal, Montréal, 148 pages.

Berthelette, D. (2002). Les déterminants de l'implantation et des résultats des programmes de santé et de sécurité du travail. In Santé, sécurité et transformation du travail, sous la direction de Denis Harrisson et Camille Legendre, Presses de l'Université du Québec, p. 107-125.

Bourgeois, F., LeMarchand, C., Hubault, F., Brun, C., Polin, A. et Faucheux, J.M. (2000). Troubles musculo-squelettiques et travail. Quand la santé interroge l'organisation. Éditions Anact, 252 pages.

Bourgeois, F., Kemarchand, C., Hubault, F., Brun, C., Polin, A. et Faucheux, J.M. (2006). Troubles musculo-squelettiques et travail. Quand la santé interroge l'organisation. (Collection Outils et méthodes), Anact, 308 pages.

Brousselle, A., Champagne, F., Contandriopoulos, A.-P. et Hartz, Z. (2009). L'évaluation : concepts et méthodes, Les Presses de l'Université de Montréal, 300 pages. 
Burgess, R.G. (1986). Approaches to field research. In Field research: a Sourcebook and Field manual. Edited by Robert G. Burgess. Contemporary Social Research. Taylor \& Francis Group, p. 1-19.

Caroly, S. (2001). Régulations individuelles et collectives de situations critiques dans un secteur de service : le guichet de la Poste. Thèse de doctorat d'ergonomie, École pratique des hautes études, Paris, 305 p. plus annexes.

Caroly, S. (2010). L'activité collective et la réélaboration des règles : des enjeux pour la santé au travail. Habilitation à diriger des recherches, Université Victor Segalen Bordeaux 2, 260 pages.

Centre National de Ressources Textuelles et Lexicales, 2010, www.cnrtl.fr.

Chassaing, K. (2006). Élaboration, structuration et réalisation des gestuelles de travail : les gestes dans l'assemblage automobile et dans le coffrage des ponts d'autoroute. Thèse de doctorat, Paris, Conservatoire national des arts et métiers, $279 \mathrm{p}$.

Chatigny, C., Vézina, N. et Prévost, J. (2003). Formation et soutien à l'apprentissage : des conditions indispensables à la polyvalence et à la santé et sécurité au travail. PISTES, vol. $5, \mathrm{n}^{\circ} 2$, 20 pages. www.pistes.uqam.ca/v5n2/pdf/v5n2a6.pdf.

Chiang, H., Ko, Y., Chen, S., Yu, H., Wu, T., Chang, P. (1993). Prevalence of shoulder and upperlimb disorders among workers in the fish-processing industry. Scandinavian Journal of Work and Environmental Health, vol. 19, p. 126-131.

Chrétien, P. (2006). Quebec experience on diagnosis and management of WMSDs in crab plants. SafetyNet-CARWH International Conference. Research on Workplace Health and Safety: From the Core to the Margins. June 7-10, 2006. St. John's, Newfoundland.

Clot, Y., Faïta, D., Fernandez, G. et Scheller, L. (2000). Entretiens en autoconfrontation croisée : une méthode en clinique de l'activité. PISTES, vol. 2, nº 1, 7 pages. www.pistes.uqam.ca/v2n1/ pdf/v2n1a3.pdf.

Cloutier, E. (1994). The effect of age on safety work practices among domestic trash collectors in Québec. Safety Science, 17, 291-308.

Cloutier, E., David, H., Ledoux, E., Bourdouxhe, M., Teiger, C., Gagnon, I. et Ouellet, F. (2005). Importance de l'organisation du travail comme soutien aux stratégies protectrices des auxiliaires familiales et sociales et des infirmières des services de soins et de maintien à domicile. IRSST, Études et recherches, Rapport R-429, 261 pages, Montréal.

Collerette, P. (2004). Méthodes des études de cas. Dictionnaire des méthodes qualitatives en sciences humaines et sociales ( $2^{\mathrm{e}}$ édition). Paris : Armand Colin, p. 92-95.

Contandriopoulos, A.P., Champagne, F., Potvin, L., Denis, J.L. et Boyle, P. (1990). Savoir préparer une recherche : la définir, la structurer, la financer. Montréal : Les Presses de l'Université de Montréal.

Coutarel, F., Daniellou, F., Dugué, B. (2003). Interroger l'organisation du travail au regard des marges de manoeuvre en conception et en fonctionnement : la rotation est-elle une solution aux TMS ? PISTES, vol. 5, $\mathrm{n}^{\circ}$ 2, 24 pages. www.pistes.uqam.ca/v5n2/pdf/v5n2a2.pdf

Curie, J. et Hajjar, V. (1987). Vie de travail - Vie hors travail : la vie en temps partagé. In LévyLeboyer, C. et Sperandio, J.C. (Eds.). Traité de psychologie du travail. Paris: PUF, p. 37-55.

Daniellou, F. (2005). The French-speaking ergonomists' approach to work activity: crossinfluences of field intervention and conceptual models. Theoretical Issues in Ergonomics Science, vol. $6, n^{\circ} 5$, p. 409-427. 
De La Garza, C. et Weill-Fassina, A. (2000). Régulations horizontales et verticales du risque. In Weill-Fassina, A., Hakim Benchekroun, T. Le travail collectif : perspectives actuelles en ergonomie. Toulouse : Octarès Éditions, p. 217-234.

Denis, D., St-Vincent, M., Gonella, M., Couturier, F. et Trudeau, R. (2007). Analyse des stratégies de manutention chez des éboueurs au Québec - Pistes de réflexions pour une formation. Études et recherches, IRSST, R-527, Montréal, 80 pages.

Denis, J.L., Hébert, Y., Langley, A., Lozeau, D. et Trottier, L.H. (2002). Explaining diffusion patterns for complex health care innovations. Health Care Management Review, vol. 27, n 3, p. 60-73.

Dictionnaire de la langue française. (2010). www.linternaute.com/dictionnaire/fr/definition/ strategie

Dopson, S. (2003). The potential of the case study method for organisational analysis. Policy \& Politics, vol. $31, \mathrm{n}^{\circ}$ 2, p. 217-226.

Durand, M.J., Berthelette, D., Loisel, P., Beaudet, J. et Imbeau, D. (2007). Travailleurs de la construction ayant une dorso-lombalgie. Évaluation de l'implantation d'un programme de collaboration précoce en réadaptation. Rapport IRSST R-489, Montréal, 92 pages.

Durand, M.J., Vézina, N., Baril, R., Loisel, P., Richard, M.C. et Ngomo, S. (2008). La marge de manœuvre de travailleurs pendant et après un programme de retour progressif au travail. Rapport IRSST R-566, Montréal, 58 pages.

Eisenhardt, R.M. (1989). Building theories from case study research. Academy of Management Review, vol. 14, $\mathrm{n}^{\circ}$ 4, p. 532-550.

Gaudart, C. (1996). Transformations de l'activité avec l'âge dans des tâches de montage automobile sur chaîne. Thèse de doctorat en ergonomie. École Pratique des Hautes Études, Paris, $215 \mathrm{p}$.

Gautrin, D., Cartier, A., Howse, D., Horth-Susin, L., Jong, M., Swanson, M., Lehrer, S., Fox, G. et Neis, B. (2010). Occupational asthma and allergy in snow crab processing in Newfoundland and Labrador. Occupational and Environmental Medicine, vol. 67, nº 1, p. 17-23.

Gonon, O. (2003). Des régulations en lien avec l'âge, la santé et les caractéristiques du travail : le cas des infirmières d'un centre hospitalier français. PISTES, vol. $5, \mathrm{n}^{\circ} 1,17$ pages, www.pistes.uqam.ca/v5n1/articles/v5n1a1.htm

González, R. et Weill-Fassina, A. (2005). Modalités de régulation du processus de travail dans les activités de service en crèche. @ctivités, vol. 2, nº 2, p. 2-23, www.activites.org/v2n2/ gonzales.pdf.

Guérin, F., Laville, A., Daniellou, F., Duraffourg, J. et Kergulen, A. (2006). Comprendre le travail pour le transformer. La pratique de l'ergonomie. Anact, 318 pages.

Howse, D. ; Gautrin, D. ; Neis, B. ; Cartier, A. ; Horth-Susin, L. ; Jong, M. et Swanson, M. C. (2006). Gender and snow crab occupational asthma in Newfoundland and Labrador, Canada. Environmental Research, vol. 101, n 2, p. 163-174.

Laperrière, A. (1997). Les critères de scientificité des méthodes qualitatives. In La recherche qualitative : enjeux épistémologiques et méthodologiques. Boucherville : Gaëtan Morin éditeur, p. 365-389.

Laville A., Teiger C. et Duraffourg J. (1972). Conséquences du travail répétitif sous cadence sur la santé des travailleurs et les accidents. Paris : Collection du Laboratoire de Physiologie du travailErgonomie du Conservatoire National des Arts et Métiers, rapport final n ${ }^{\circ} 29,385$. 
Magnier, J. et Werthe, C. (2001). L'expérience revisitée à l'occasion de la validation des acquis professionnels. Formation Emploi, $\mathrm{n}^{\circ}$ 75, p. 29-41.

Major, M.E. et Vézina, N. (2007). Follow up of the evolution of seasonal workers' pain and musculoskeletal symptoms in the snow crab processing industry. Sixth International Scientific Conference on Prevention of Work-Related Musculoskeletal Disorders PREMUS, 28 août 2007, Boston, États-Unis.

Major, M.E. (2005). Étude ergonomique du travail saisonnier et de ses impacts sur les stratégies et les troubles musculo-squelettiques de travailleuses d'usines de transformation du crabe. Proposition de recherche présentée comme exigence partielle du doctorat en sciences biologiques, UQAM, 59 pages.

Major, M.E. (2011). Étude ergonomique du travail saisonnier et de ses impacts sur les stratégies et les troubles musculo-squelettiques de travailleuses d'usines de transformation du crabe. Thèse de doctorat en sciences biologiques, UQAM.

Malo, J., Chrétien, P., McCants, M., Lehrer, S. (1997). Detection of snow crab antigens by air sampling of a snow crab production plant. Clinical \& Experimental Allergy, vol. 27, $\mathrm{n}^{\circ}$ 1, p. 75-78.

Malo, J. (1992). Occupational lung disease and compensation issues. Med. North Am., p. 3736-3739.

Malo, J.-L., Cartier, A., Gezzo, H., Lafrance, M., McCants, M., Lehrer, S.B. (1988). Patterns of improvement in spirometry, bronchial hyperresponsiveness, and specific IgE antibody levels after cessation of exposure in occupational asthma caused by snowcrab processing. American Review of Respiratory Disease, vol. 138, p. 807-812.

Mergler, D., Vézina, N., Beauvais, A., Everell, J. (1983). The effects of working conditions on the health of slaughterhouse workers. Canadian Center for Occupational Health and Safety, Hamilton, Report 047.

Messing, K. et Reveret, J.P. (1983). Are women in female jobs for their health? A study of working conditions and health effects in the fish-processing industry in Quebec. International Journal of Health Services, vol. 13, $\mathrm{n}^{\circ}$ 4, p. 635-647.

Miles, M.B. et Huberman, A.M. (2003). Analyse des données qualitatives. Bruxelles : De Boeck Université. 626 pages.

Mongeau, P. (2008). Réaliser son mémoire ou sa thèse. Côté Jeans et côté Tenue de soirée. Presses de l'Université du Québec, Québec, 145 pages.

Mucchielli, R. (1996). Dictionnaire des méthodes qualitatives en sciences humaines et sociales. Paris : Armand Colin.

Mucchielli, R. (2006). L'analyse de contenu (9e édition). Paris : Les éditions ESF.

Neis, B. (1994). Santé et sécurité au travail des travailleuses de l'industrie de la transformation du poisson et du crabe de Terre-Neuve et du Labrador. Maladies chroniques au Canada, vol. $15, \mathrm{n}^{\circ} 1$, p. 6-11.

Neis, B. et Grzetic, B. (2001). Restructuration et santé des femmes : la crise de la pêche à TerreNeuve. Bulletin de recherche des centres d'excellence pour la santé des femmes, vol. 2, nº 2, p. 14-17.

Nordander, C., Ohlsson, K., Balogh, I., Rylander, L., Palsson, B., Skerfving, S. (1999). Fish processing work: The impact of two sex dependent exposure profiles on musculoskeletal health. Occupational and Environmental Health, vol. 56, p. 256-264. 
Ohlsson, K., Hansson, G.A., Balogh, I., Stromberg, U., Palsson, B., Nordander, C., Rylander, L. et Skerfving, S. (1994). Disorders of the neck and upper limbs in women in the fish processing industry. Occupational and Environmental Medicine, vol. 51, $\mathrm{n}^{\circ} 12$, p. 826-832.

Ouellet, S. et Vézina, N. (2008). Savoirs professionnels et prévention des TMS : réflexions conceptuelles et méthodologiques menant à leur identification et à la genèse de leur construction. PISTES, vol. 10, no.2, 35 pages. www.pistes.uqam.ca/v10n2/pdf/v10n2a5.pdf

Ouellet, S. (2009). Acquisition d'habiletés motrices à la découpe de viande et prévention des troubles musculo-squelettiques : apport de l'analyse ergonomique à la conception de formations. Thèse de doctorat, Montréal, UQAM.

Organisation mondiale de la Santé. Préambule à la Constitution de l'Organisation mondiale de la Santé, Conférence internationale sur la santé, New York, 19-22 juin 1946 ; signée le 22 juillet 1946 par les représentants de 61 États. 1946 ; (Actes officiels de l'Organisation mondiale de la Santé, $\mathrm{n}$ ○ 2, p. 100) et entrée en vigueur le 7 avril 1948. La définition du terme «santé » n'a pas été modifiée depuis 1946 : www.who.int/about/definition/fr/print.html

Premji, S., Lippel, K. et Messing, K. (2008). « On travaille à la seconde ! » Rémunération à la pièce et santé et sécurité du travail dans une perspective qui tient compte de l'ethnicité et du genre. PISTES, vol. 10, $\mathrm{n}^{\circ} 1$, p. 1-24.

Prévost, J. et Messing, K. (2001). Stratégies de conciliation d'un horaire de travail variable avec des responsabilités familiales. Le Travail Humain, vol. 64, $\mathrm{n}^{\circ}$ 2, p. 119-143.

Pueyo, V. et Gaudart, C. (2000). L'expérience dans les régulations individuelles et collectives des déficiences. Le Travail Collectif. Perspectives actuelles en ergonomie. In T.H. Benchekroun et A. WeillFassina (Eds.). Toulouse: Octarès Éditions, p. 257-272.

Quinlan, M. (2010). Recent research into the health, safety and related regulatory effects of precarious employment. Conférence IRSST, 28 septembre 2010, Montréal, Québec.

Richard, J.G. (1997). Identification des outils requis pour accroître l'efficacité des stratégies de prévention des lésions attribuables au travail répétitif dans les abattoirs et usines de transformation. Rapport IRSST R-158, 81 pages.

Savoie-Zajc, L. (1997). L'entrevue semi-dirigée. Dans B. Gauthier (Ed.). Recherche sociale : de la problématique à la collecte des données. $3^{\mathrm{e}}$ éd. Presses de l'Université du Québec, p. 263-285.

Savoie-Zajc, L. (2003). L'entrevue semi-dirigée. Dans B. Gauthier (Ed.). Recherche en sciences sociales : de la problématique à la collecte des données. 4 éd. Ste-Foy: PUQ. p. 293-316.

Schrank, W.E. (2005). The Newfoundland fishery: ten years after the moratorium. Marine Policy, vol. 29, p. 407-420.

Schweder, P. (2008). Occupational health and safety of seasonal workers in agricultural processing. Ph.D. Thesis University of New South Wales. 274 pages.

Sieber, J.E. (1982). The ethics of social research: Fieldwork, regulation, and publication. New York Springer, 187 pages.

Solberg, S.M., Vézina, N. et Molgaard, J. (2005). Cumulative trauma disorders among crabprocessing workers. Canadian Association for Research on Work and Health Conference (CARWH), University of British Columbia, Vancouver, B.C., May 16-17, 2005.

Stake, R.E. (1995). The art of case study research. Thousand Oaks (CA): Sage Publications. 
Stock, S., Loisel, P., Durand, M.-J., Streiner, D., Tugwell, P., Reardon, R., Lemaire, J., Boucher, M., Darzins, S., Dilworth, P. et Gaudreault, N. (2003). IDVQ : L'indice d'impact de la douleur au cou et aux membres supérieurs sur la vie quotidienne. IRSST Études et recherches, Rapport R-355, 99 pages.

St-Vincent, M., Vézina, N., Bellemare, M., Denis, D., Ledoux, E. et Imbeau, D. (2011). L'intervention en ergonomie. Éditions MultiMondes, Québec, 360 pages.

Teiger, C., Cloutier, E. et David, H. (2005). Les activités de soins à domicile, soigner et prendre soin. Presses Universitaires de France (PUF), In Situations de service : travailler dans l'interaction, chapitre 5, 254 pages.

Thériault, G. (2007). Rapport sur l'industrie du crabe des neiges du Nouveau-Brunswick. GTA Consultants en pêche inc. 96 pages.

Toulouse G., Vézina G., Geoffrion L. (1995). Études descriptives des déterminants des facteurs de risque de LATR aux postes d'éviscération de deux abattoirs de porcs. (Collection Études et Recherches), IRSST, R-108.

Toupin, C. (2005). L'élaboration des stratégies de travail nocturnes : le cas d'infirmières de nuit d'un service de pneumologie français. PISTES, vol. 7, $\mathrm{n}^{\circ}$ 1, 18 pages, www.pistes.uqam.ca/v7n1/ pdf/v7n1a4.pdf.

Van der Maren, J.M. (1993). Méthodes de recherche pour l'éducation. Montréal : Librairie de l'Université de Montréal.

Vermersch, P. (1994). L'entretien d'explicitation en formation initiale et en formation continue. Paris : ESF.

Vermersch, P. (1991). L'entretien d'explicitation. Les cahiers de Beaumont, $n^{\circ}$ 52, p. 63-70.

Vermersch, P. (1989). Expliciter l'expérience. Éducation Permanente. 100-101, p. 123-132.

Vézina, N., Courville, J., Geoffrion, L. (1995). Problèmes musculo-squelettiques et caractéristiques des postes des travailleurs et des postes des travailleuses sur une même chaîne de découpe de dinde. In K. Messing, B. Neis, L. Dumais, ed., Invisible : Women's Occupational Health and Safety. Charlottetown, Gynergy Press, p. 29-61.

Vézina, N., Prévost, J., Lajoie, A. et Beauchamp, Y. (1999). Élaboration d'une formation à l'affilage des couteaux : le travail d'un collectif, travailleurs et ergonomes. PISTES, vol. 1, $\mathrm{n}^{\circ} 1$, www.pistes.uqam.ca.

Vézina, N. (2001). La pratique de l'ergonomie face aux TMS : Ouverture à l'interdisciplinarité. Comptes rendus du Congrès SELF-ACE 2001 - Les transformations du travail, enjeux pour l'ergonomie, p. 44-60.

Vézina, N., Stock, S., Simard, M., St-Jacques, Y., Marchand, A., Bilodeau, P.-P., Boucher, M., Zaabat, S. et Campi, A. (2003). Problèmes musculo-squelettiques et organisation modulaire du travail dans une usine de fabrication de bottes. Rapport R-345, Montréal, IRSST, 125 pages.

Vézina, N. (2003). La rotation, est-ce une solution ? PISTES, vol. 5, $\mathrm{n}^{\circ} 2,4$ pages. www.pistes.uqam.ca/v5n2/pdf/v5n2a10.pdf

Vézina, N., Ouellet, S. et Major, M.E. (2009). Quels schémas corporels pour la prévention des troubles musculo-squelettiques? Corps, dossier thématique Corps au travail, no. 6, Paris Éditions Dilecta.

Yin, R.K. (2003). Case Study Research: Design and Methods. $3^{\text {rd }}$ ed. Applied Social Research Methods Series. Thousand Oaks, CA : Sage, 181 pages. 


\section{ANNEXES}

Postes occupés par les travailleuses suivies

\begin{tabular}{|c|c|c|c|c|c|c|c|c|c|c|c|c|c|c|c|c|c|}
\hline & \multirow[b]{2}{*}{ Travailleuses/Postes } & \multicolumn{7}{|c|}{ Côte-Nord } & \multicolumn{8}{|c|}{ Terre-Neuve } & \multirow[b]{2}{*}{ Total } \\
\hline & & 1 & 2 & 3 & 4 & 5 & 7 & 8 & 1 & 2 & 3 & 4 & 5 & 6 & & 8 & \\
\hline \multirow{10}{*}{ Crabe } & Triage & & & & & & & & & & & & & & & $\mathrm{x}$ & 1 \\
\hline & Empaquetage & $x$ & $\mathrm{x}$ & $\mathbf{x}$ & & & & & $\mathrm{x}$ & & & & & & & & 4 \\
\hline & Empaquetage et sciage & & & & & & & & $\mathrm{x}$ & & & $\mathrm{x}$ & & & & & 2 \\
\hline & Classement des pinces & & & & & & & & & $\mathrm{x}$ & & & & & & & 1 \\
\hline & Pesage & & & & & & & & & & $\mathrm{x}$ & & & & & & 1 \\
\hline & Emballage & & & $\mathbf{x}$ & & & & & & & & & & $\mathbf{x}$ & & & 2 \\
\hline & Chariot & & & & $\mathrm{x}$ & & & & & & & & & & & & 1 \\
\hline & Laver les paniers & & & & & & & & & & & & $\mathbf{x}$ & & & & 1 \\
\hline & Faire des boîtes & & & & & & $\mathrm{x}$ & & & & & & & $\mathbf{x}$ & & & 2 \\
\hline & Mettre étampes & & & & & & $\mathrm{x}$ & & & & & & & & & & 1 \\
\hline \multirow{8}{*}{$\begin{array}{l}\text { Chair de } \\
\text { crabe }\end{array}$} & Couper pinces & & & & & $\mathbf{x}$ & $x$ & $x$ & & & & & & & $\mathbf{x}$ & & 4 \\
\hline & Casser corps et pattes & & & & & $\mathrm{x}$ & $\mathrm{x}$ & & & & & & & & & & 2 \\
\hline & Machine à pattes & & & & & $\mathrm{x}$ & $x$ & $x$ & & & & $\mathrm{x}$ & & & & & 4 \\
\hline & Vider corps & & & & & $\mathrm{x}$ & $x$ & $x$ & & & & & & & & & 3 \\
\hline & Vider pinces & & & & & $\mathrm{x}$ & & & & & & & & & & & 1 \\
\hline & Mirer chair & & & & & & $x$ & $x$ & & & & & & & & & 2 \\
\hline & Sciage pattes & & & & & & & & & & & & & & & $\mathrm{x}$ & 1 \\
\hline & Mettre chair dans boîte & & & & & $\mathrm{x}$ & & & & & & & & & $\mathrm{x}$ & & 2 \\
\hline \multirow[t]{4}{*}{ Capelans } & Triage capelans & & & & & & & & & & & & & & & & 0 \\
\hline & Empaquetage capelans & & & & & & & & & & $\mathrm{x}$ & & & $\mathbf{x}$ & & & 2 \\
\hline & Pesage capelans & & & & & & & & & & $\mathbf{x}$ & & & & & & 1 \\
\hline & Emballage capelans & & & & & & & & & & $\mathrm{x}$ & & & & & $\mathbf{x}$ & 3 \\
\hline
\end{tabular}




\begin{tabular}{|l|l|l|l|l|l|l|l|l|l|l|l|l|l|l|l|l|l|l|}
\hline $\begin{array}{l}\text { Rangement boîtes pour } \\
\text { congeler }\end{array}$ & & & & & & & & & $\mathbf{X}$ & & & & $\mathbf{1}$ \\
\hline $\begin{array}{l}\text { Emballage boîtes } \\
\text { capelans congelés }\end{array}$ & & & & & & & & $\mathbf{X}$ & & & & $\mathbf{X}$ & $\mathbf{2}$ \\
\hline & Turbot & & & & & & & & & & $\mathbf{X}$ & & & & & $\mathbf{1}$ \\
\hline & Nb postes total & $\mathbf{1}$ & $\mathbf{1}$ & $\mathbf{2}$ & $\mathbf{1}$ & $\mathbf{6}$ & $\mathbf{2}$ & $\mathbf{5}$ & $\mathbf{4}$ & $\mathbf{2}$ & $\mathbf{4}$ & $\mathbf{5}$ & $\mathbf{2}$ & $\mathbf{1}$ & $\mathbf{3}$ & $\mathbf{2}$ & $\mathbf{4}$ & $\mathbf{4 5}$ \\
\hline
\end{tabular}

\section{RÉSUMÉS}

L'étude des stratégies mises en œuvre par les travailleurs-euses est partie intégrante de l'analyse de l'activité de travail en ergonomie. Cette étude propose un cadre de référence pour le recueil et l'analyse des stratégies et décrit la nature et les enjeux des stratégies développées par des travailleuses saisonnières de l'industrie de la transformation du crabe aux prises avec des troubles musculo-squelettiques. Reposant sur un devis méthodologique combinant l'approche centrée sur l'analyse de l'activité de travail et celle d'une étude de cas multiples de 16 travailleuses (8 à Terre-Neuve; 8 au Québec) suivies pendant deux années consécutives, divers entretiens et des observations de l'activité et de l'organisation du travail ont été réalisés. Les résultats permettent de constater que les travailleuses élaborent une grande variété de types de stratégies pour gérer leur douleur (19 catégories de stratégies). On constate autant les bienfaits associés à certaines stratégies que les compromis coûteux que représentent d'autres stratégies pour la santé. Le cadre de référence élaboré de manière inductive et ancré empiriquement dans les données recueillies sur le terrain constitue un outil dont pourront disposer les chercheurs et les ergonomes pour l'identification et le classement des stratégies.

Study of the strategies used by workers is a central aspect of work activity analysis in ergonomics. This study proposes a conceptual framework for strategy collection and analysis, and describes the nature and issues of the strategies developed by seasonal crab plant workers with musculoskeletal disorders. Based on a methodology combining the ergonomic approach of work activity analysis and a multiple case study of 16 female workers ( 8 in Newfoundland, 8 in Quebec), interviews and observations of the work activity and organization were conducted for two consecutive years. While showing a wide range of strategies developed by workers to manage pain (19 categories of strategies obtained), the results also showed the advantages of some strategies and the costly health compromises of other strategies. The inductively developed framework, based empirically on field data, is a tool that can be used by researchers and ergonomists to identify and classify strategies.

El estudio de las estrategias aplicadas por los trabajadores-as es parte integrante del análisis de la actividad de trabajo en ergonomía. Este estudio propone un marco de referencia para la colecta y el análisis de las estrategias y describe su naturaleza y lo que esta en juego en las estrategias desarrolladas por las trabajadoras temporarias de la industria de tratamiento del cangrejo que sufren problemas musculo-esqueléticos. Se realizaron diversas entrevistas y observaciones de la actividad de trabajo y de la organización de trabajo, apoyadas en una aproximación metodológica que combina un enfoque centrado en el análisis de la actividad de trabajo y un estudio de casos múltiples de 16 trabajadoras (8 en Terra-Nova; 8 en Québec), seguidas durante dos años consecutivos. Los resultados permiten constatar que las trabajadoras elaboran una gran variedad de tipos de estrategias para manejar su dolor (19 categorías de estrategias). Constatamos tanto 
los beneficios asociados a ciertas estrategias como los compromisos costosos para la salud que representan otras estrategias. El marco de referencia elaborado de manera inductiva y anclado empíricamente en los datos colectados en el terreno, constituye una herramienta que podrá ser utilizada por los investigadores y los ergónomos para la identificación y la clasificación de las estrategias.

INDEX

Palabras claves : estrategias, regulación, análisis de la actividad, estudio de casos múltiples, problemas musculo-esqueléticos

Keywords : strategies, regulation, work activity analysis, case study, musculoskeletal disorders Mots-clés : stratégies, régulation, analyse de l'activité, étude de cas multiples, troubles musculosquelettiques

\section{AUTEURS}

\section{MARIE-ÈVE MAJOR}

major.marie-eve@.uqam.ca, CINBIOSE, Université du Québec à Montréal, C.P. 8888, succursale Centre-ville, Montréal (Québec), H3C 3P8

\section{NICOLE VÉZINA}

vezina.nicole@uqam.ca, CINBIOSE, Université du Québec à Montréal, C.P. 8888, succursale Centre-ville, Montréal (Québec), H3C 3P8 